\author{
SANDIA REPORT \\ SAND2007-5042 \\ Unlimited Release \\ Printed August 2007
}

\title{
Anatomy of a SAR Impulse Response
}

\author{
Armin W. Doerry
}

Prepared by

Sandia National Laboratories

Albuquerque, New Mexico 87185 and Livermore, California 94550

Sandia is a multiprogram laboratory operated by Sandia Corporation,

a Lockheed Martin Company, for the United States Department of Energy's

National Nuclear Security Administration under Contract DE-AC04-94AL85000.

Approved for public release; further dissemination unlimited.

\section{Sandia National Laboratories}


Issued by Sandia National Laboratories, operated for the United States Department of Energy by Sandia Corporation.

NOTICE: This report was prepared as an account of work sponsored by an agency of the United States Government. Neither the United States Government, nor any agency thereof, nor any of their employees, nor any of their contractors, subcontractors, or their employees, make any warranty, express or implied, or assume any legal liability or responsibility for the accuracy, completeness, or usefulness of any information, apparatus, product, or process disclosed, or represent that its use would not infringe privately owned rights. Reference herein to any specific commercial product, process, or service by trade name, trademark, manufacturer, or otherwise, does not necessarily constitute or imply its endorsement, recommendation, or favoring by the United States Government, any agency thereof, or any of their contractors or subcontractors. The views and opinions expressed herein do not necessarily state or reflect those of the United States Government, any agency thereof, or any of their contractors.

Printed in the United States of America. This report has been reproduced directly from the best available copy.

Available to DOE and DOE contractors from

U.S. Department of Energy

Office of Scientific and Technical Information

P.O. Box 62

Oak Ridge, TN 37831

Telephone: $\quad$ (865) 576-8401

Facsimile: (865) 576-5728

E-Mail: reports@adonis.osti.gov

Online ordering: http://www.osti.gov/bridge

Available to the public from

U.S. Department of Commerce

National Technical Information Service

5285 Port Royal Rd.

Springfield, VA 22161

Telephone: $\quad$ (800) 553-6847

Facsimile: (703) 605-6900

E-Mail: $\quad$ orders@ntis.fedworld.gov

Online order: $\quad$ http://www.ntis.gov/help/ordermethods.asp?loc=7-4-0\#online

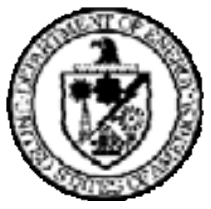


SAND2007-5042

Unlimited Release

Printed August 2007

\title{
Anatomy of a SAR Impulse Response
}

\author{
Armin W. Doerry \\ SAR Applications Department \\ Sandia National Laboratories \\ PO Box 5800 \\ Albuquerque, NM 87185-1330
}

\begin{abstract}
A principal measure of Synthetic Aperture Radar (SAR) image quality is the manifestation in the SAR image of a spatial impulse, that is, the SAR's Impulse Response (IPR). IPR requirements direct certain design decisions in a SAR. Anomalies in the IPR can point to specific anomalous behavior in the radar's hardware and/or software.
\end{abstract}




\section{ACKNOWLEDGEMENTS}

This work was funded by the US DOE Office of Nonproliferation \& National Security, Office of Research and Development, NA-22, under the Advanced Radar System (ARS) project.

Sandia is a multiprogram laboratory operated by Sandia Corporation, a Lockheed Martin Company, for the United States Department of Energy under Contract DE-AC0494AL85000. 


\section{CONTENTS}

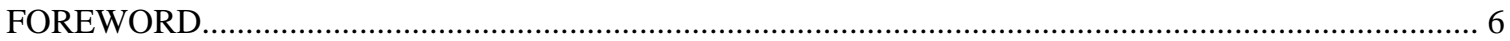

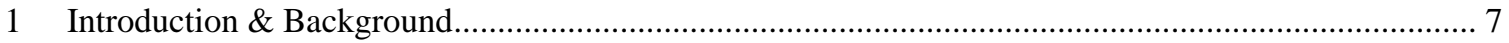

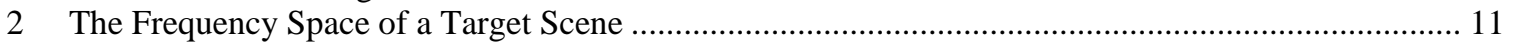

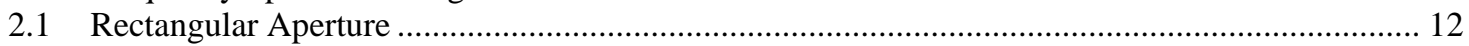

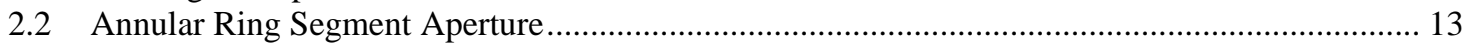

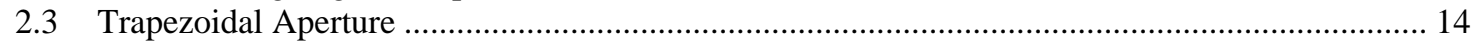

3 Sidelobe Control of the IPR ……………………….............................................................. 15

3.1 Rectangular Aperture - Windowed...................................................................................... 16

3.2 Annular Ring Segment Aperture - Windowed ........................................................................... 17

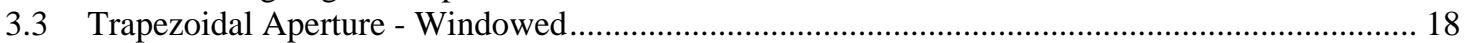

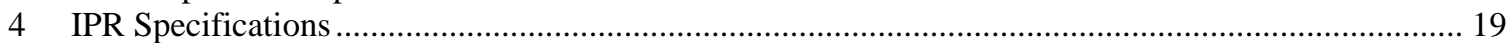

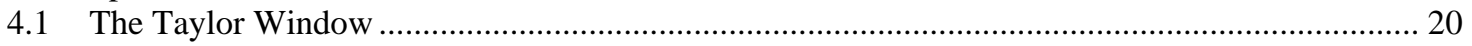

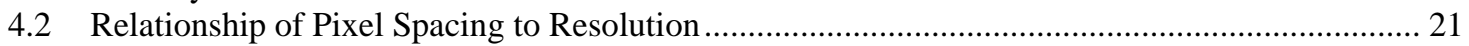

5 Effects of Non-Ideal Radar Performance on IPR ……..................................................................... 25

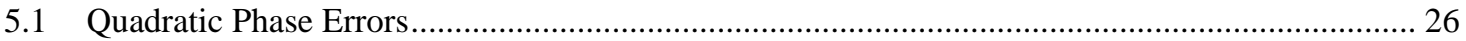

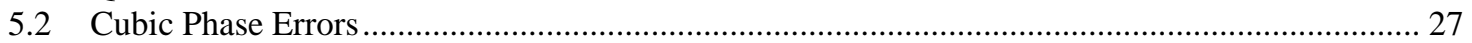

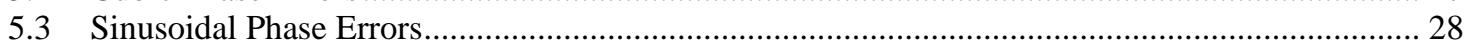

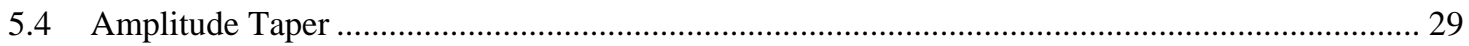

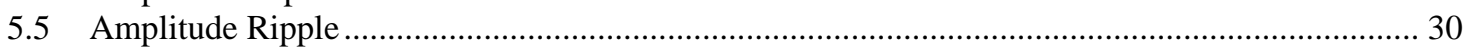

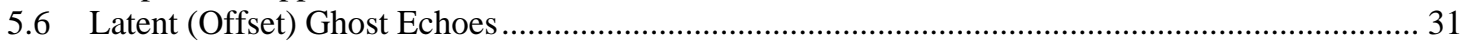

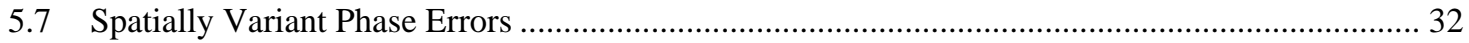

5.8 Excessive Amplitude.......................................................................................................... 33

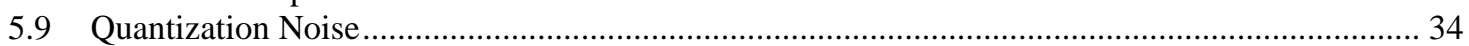

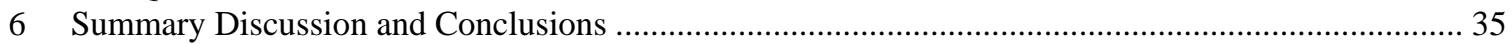

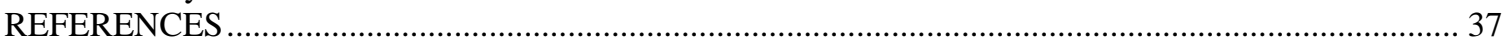

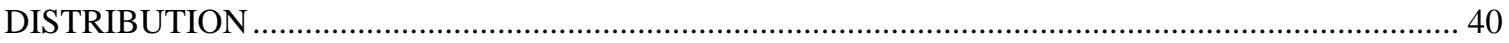




\section{FOREWORD}

The principal measure of goodness for a Synthetic Aperture Radar (SAR) system is its Impulse Response (IPR). Often, the first indication of improperly functioning SAR is when its IPR fails to meet its specification. The characteristics of the IPR can tell much about the state of health of a SAR system. Consequently, the SAR system engineer needs to have a good grasp of what constitutes a good IPR, and reading it for specific anomalous hardware and/or software operation.

The intent of this report is to catalog IPR characteristics and common anomalies for SAR systems in general, and Sandia designed SAR systems specifically. 


\section{Introduction \& Background}

The typical output of a Synthetic Aperture Radar (SAR) is an image. This image is a rendering of the radar reflectivity of a target scene. As a system concept, this is illustrated in Figure 1. A reasonable question to ask is "How faithful is the rendering (i.e. image) to the target scene?” The fidelity of the rendering with respect to the target scene is dependent on the operating capabilities of the radar hardware and signal processing. This includes bandwidths, both microwave and Doppler, and the linearity of the signal processing, both analog and digital.

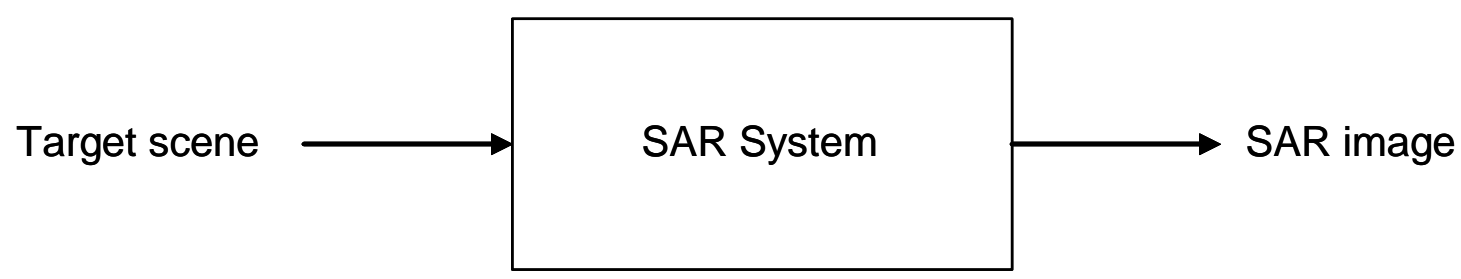

Figure 1. A SAR system operates on a target scene to produce an image.

A measure of the fidelity of the image with respect to the target scene requires that we in fact have 'truth' to measure against. The simplest truth target is one of infinitesimally small size in all dimensions, but with enough Radar Cross Section (RCS) to be clearly observable in the image, and furthermore with enough RCS to reveal non-ideal radar characteristics. Furthermore yet, such a target ideally is distortionless, that is, it has flat frequency response and linear phase across the bandwidth of the radar. Gain or loss may be arbitrary, but must be constant over frequency. This causes range to be directly proportional to delay, the virtually uniform assumption of all radars. Such a target is referred to as a "point target", "impulse reflector", or "impulse target" due to its spatial characteristics and interaction with radar waveforms resembling a mathematical impulse or Dirac delta function.

Of course a physical measurement requires a real target, not just a hypothetical construct. Canonical targets such as trihedrals, tophats, and spheres can often mimic hypothetical point reflectors adequately enough for useful radar characterization.

The response to a hypothetical spatial impulse target is then the Impulse Response (IPR) of the radar. In SAR, which offers a two-dimensional image, the IPR is a twodimensional entity. Figure 2 illustrates the system model that corresponds to Figure 1.

The radar interrogates a target scene by transmitting pulses and receiving and processing reflections or echoes. Echo timing corresponds to spatial locations. This is how the radar measures the spatial content of a target scene, including any spatial impulse targets. 
Consequently, time-domain characteristics of the radar influence the ability of the radar to measure spatial locations, i.e. the radar IPR. Of course, time-domain characteristics have unique frequency-domain counterparts. Consequently the frequency-domain characteristics of the radar equivalently influence the ability of the radar to measure spatial locations, i.e. the radar IPR.

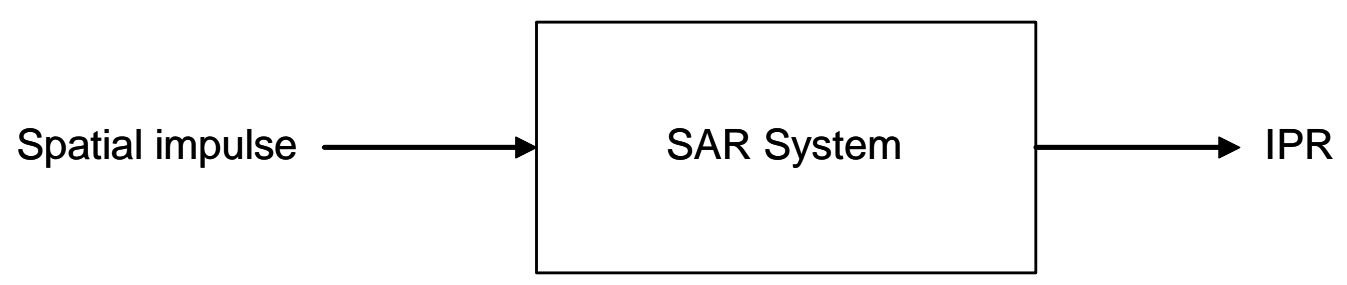

Figure 2. A spatial impulse target produces an Impulse Response in the image.

We reiterate that the point target, in order to be a point target, needs to exhibit linear phase (constant group delay) over the frequencies used by the radar, that is, without dispersion.

In order to most accurately render the target scene, the SAR system tries to render a spatial impulse at its input (see Figure 2) to as near a spatial impulse at its output as possible. So the ideal IPR is also an impulse, or at least as near to an impulse as the radar is capable of producing. As such, the SAR system itself tries to be as distortionless as possible, that is, with constant (flat) passband and linear phase. The reality is that the passband is generally band-limited, both in frequency and in Doppler. This constrains how accurately a spatial impulse can be reproduced at the output, that is, the shape of the IPR. This is illustrated in Figure 3 and Figure 4. Furthermore, as we shall see, accuracy in the mean-squared-error sense might also be sacrificed somewhat at times to deal with other undesirable effects of being band limited, namely sidelobes in the IPR as illustrated in Figure 4. ${ }^{1}$

Since a perfect point target offers only delay and attenuation, any transmitted waveform will also be the received waveform albeit attenuated and delayed. Consequently, the IPR also represents the SAR receiver's response when matched to the transmitted waveform. That is, the SAR IPR is a matched filter output of the radar receiver to the transmitted waveform and pulse train. A discussion of SAR matched filter characteristics can be found in other reports. ${ }^{2,3}$ We will hereafter presume a Linear-FM (LFM) waveform with its attendant nearly rectangular energy spectrum.

Finally, we note that the IPR is a two-dimensional entity, generally with axes corresponding to range and cross-range (due to Doppler, in the azimuth direction). Often, however, only principal-axes cuts are displayed. These are usually sufficient for radar characterization and ascertaining most problematic behaviors. These principal cuts are often referred to as the 'range IPR' and the 'azimuth IPR'. 


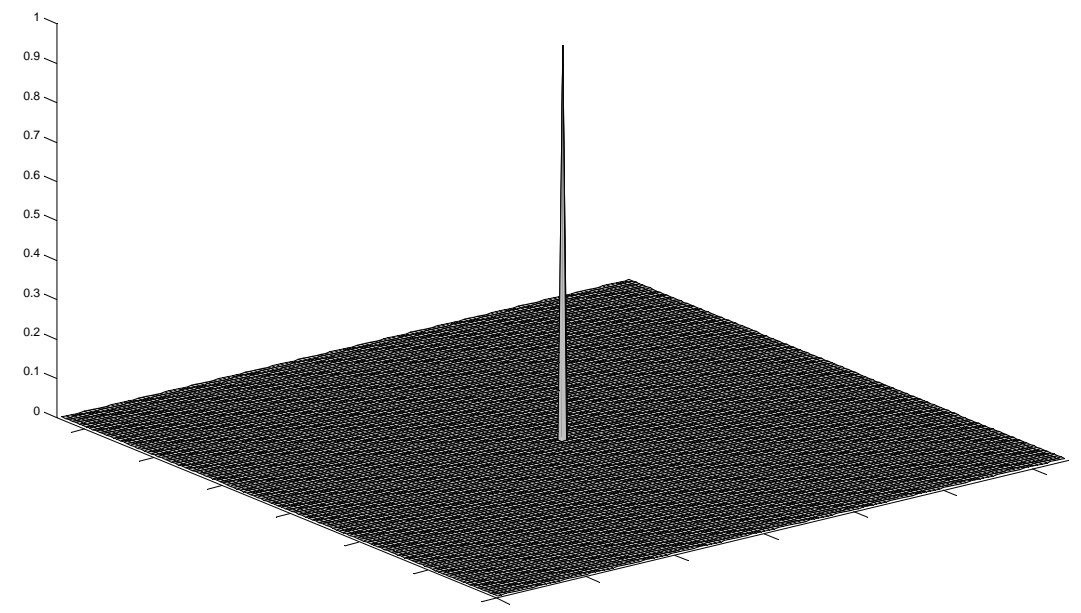

Figure 3. Example spatial impulse.

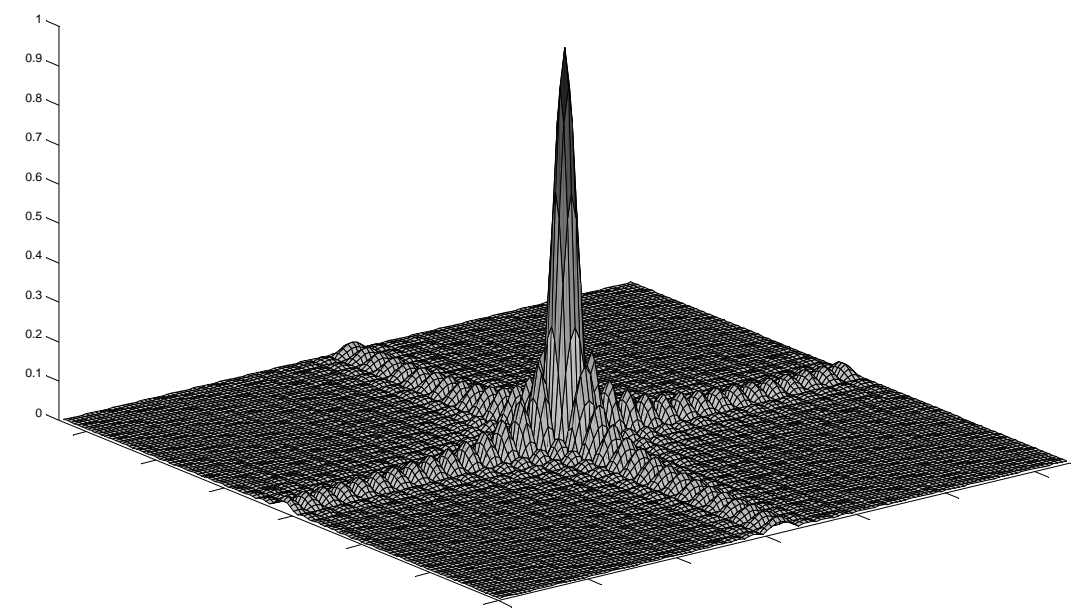

Figure 4. Band-limited IPR for spatial impulse of Figure 3. 
It is difficult to appreciate the details of IPR analysis without a good understanding of Fourier analysis. A good text in this area is by Bracewell. ${ }^{4}$ However, with such an appreciation, the relationship of a SAR's IPR to other technologies becomes readily apparent.

Raw SAR data can be manipulated to represent a surface segment in the Fourier (frequency) space of the target scene being imaged. ${ }^{5}$ This data is normally projected to two dimensions before processing, and consequently then represents a region in a twodimensional frequency plane. We refer to this projected region as the data 'aperture'.

\section{Relationship to Antenna Performance}

In antenna analysis, the far-field antenna pattern is calculated via the Fourier transform of the real antenna's aperture illumination function. This relationship is discussed by Skolnik. ${ }^{6}$ The SAR's data aperture corresponds to a real antenna's physical aperture illumination function. Consequently, the SAR's IPR then corresponds to the real antenna's far-field two-dimensional beam pattern.

\section{$\underline{\text { Relationship to Optics }}$}

In optics, the focal point of a lens yields the Fourier transform of the incident energy that it focuses. A classic reference for Fourier optics is by Goodman. ${ }^{7}$ The SAR's data aperture corresponds to a collimated incident beam (two-dimensional intensity profile) impinging on a lens. Consequently the SAR's IPR then corresponds to the Point Spread Function (PSF) at the focal point of the lens.

In fact, Dickey ${ }^{8}$ has shown how optical models and techniques yield very SAR-like images.

\section{Prior Work}

An earlier report by Cordaro $^{9}$ details aspects of LFM chirp processing for radar signals, and the impact of several kinds of signal anomalies. 


\section{The Frequency Space of a Target Scene}

As previously stated, raw SAR data can be manipulated to represent a surface segment in the Fourier (frequency) space of the target scene being imaged. This surface is generally not flat, with characteristics corresponding to the data collection geometry. ${ }^{10}$ Therefore, this surface segment is usually effectively projected onto a flat two-dimensional surface for easier processing into a two-dimensional image. In any case, the SAR data represents only a small finite portion of the Fourier space of the scene, as is illustrated in Figure 5. This finite extent is what causes sidelobes in the image.

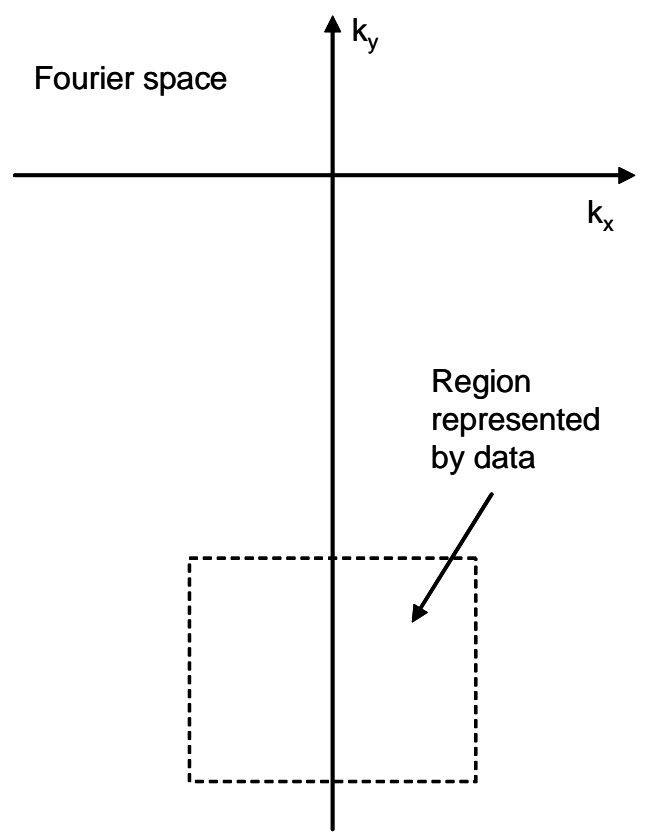

Figure 5. SAR data represents a finite neighborhood in the Fourier space of the target scene.

The extent of the data region, or 'aperture', is inversely proportional to resolution in the corresponding directions. Although Figure 5 shows a rectangular aperture, the shape of the totality of the raw SAR data region is generally not rectangular. For coarse resolutions it may be, and often is approximated as rectangular. At finer resolutions the non-rectangular nature has to be dealt with in the signal processing. Often, the Fourierspace data is cropped and resampled to a rectangular aperture with rectangular data spacing to facilitate easier image formation. Sometimes, however, it is not cropped to a rectangular aperture. This becomes observable in the sidelobe structure of the IPR.

Sidelobes are generated by the finite extent of the aperture. Consequently, in a twodimensional image, sidelobes are most obvious in directions orthogonal to the edges of the aperture. We now examine several common apertures used by various SAR systems. 


\subsection{Rectangular Aperture}

Coarse resolution SAR systems can often have their data presumed to correspond to a rectangular aperture. Finer resolution systems will often crop their data set to correspond to a rectangular aperture. This accounts for the "+" nature of the sidelobe structure.

The equal weighting of all parts of the aperture cause cardinal axes to exhibit sidelobes at $-13 \mathrm{dBc}$.
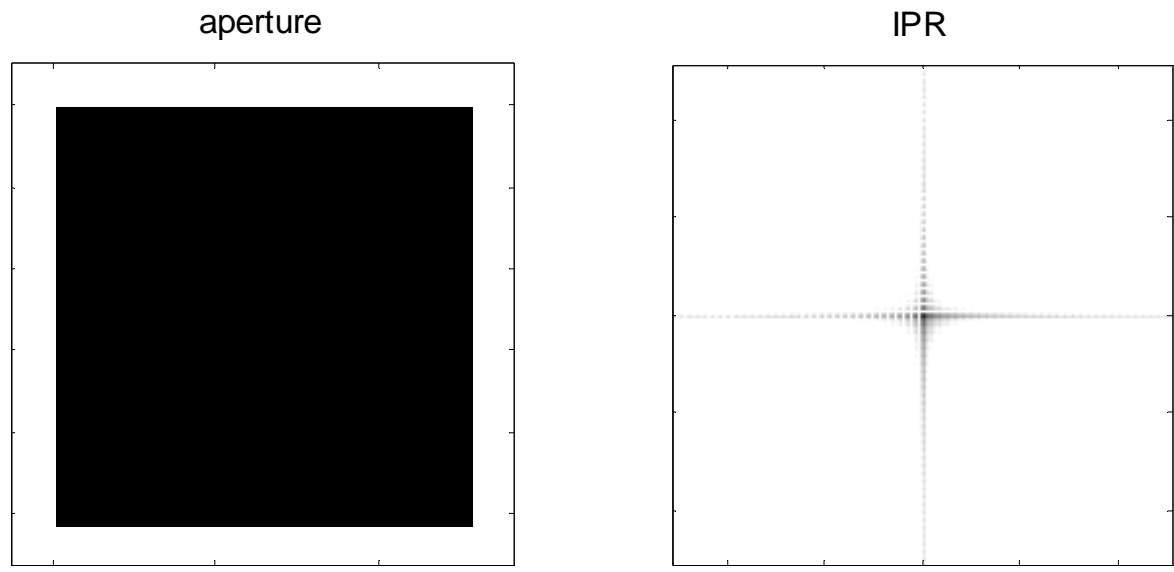

Figure 6. Rectangular aperture and corresponding IPR.
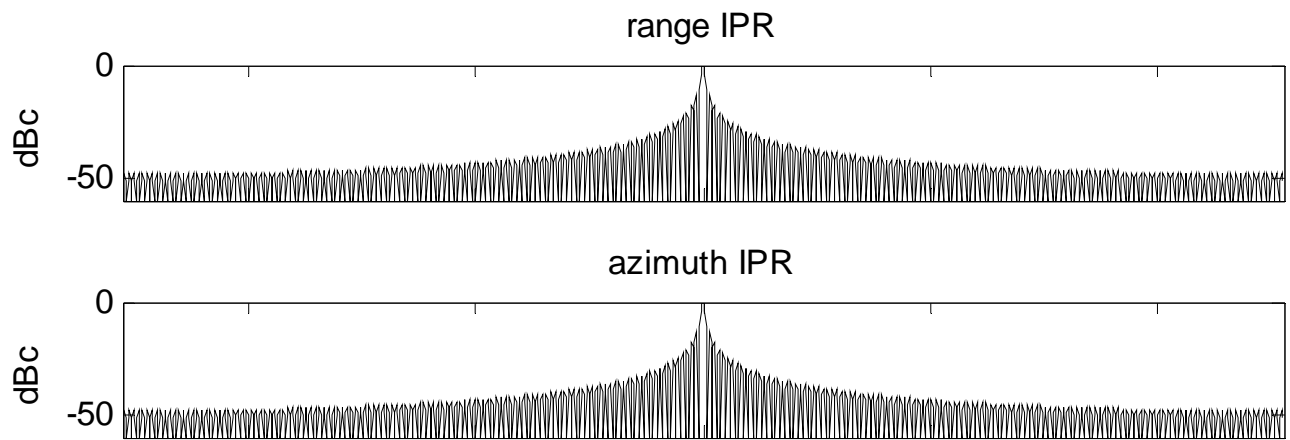

Figure 7. Principal axes cuts of the IPR. 


\subsection{Annular Ring Segment Aperture}

SAR systems with fixed constant waveform parameters that collect viewing broadside while flying in a straight line will have data corresponding to an aperture shaped as a segment of an annular ring. Often, this will be cropped to a rectangle before processing. Otherwise, the sidelobe structure would remain as in the following figures. The angle between the azimuth 'whiskers' is equal to the angle subtended during data collection. Note the fuzzy range sidelobes due to the curved upper and lower edges of the aperture.
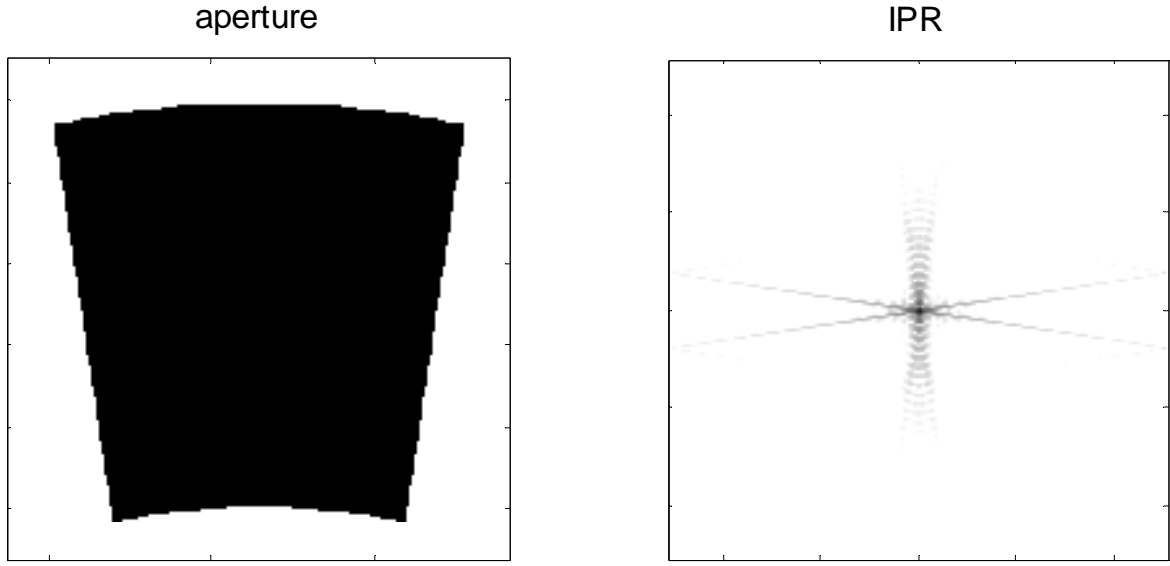

Figure 8. Annular ring segment aperture and corresponding IPR. The annular ring segment aperture corresponds to $0.025 \mathrm{~m}$ resolution

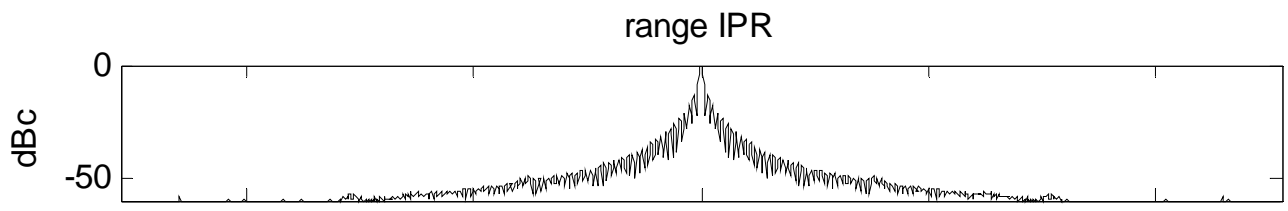

azimuth IPR

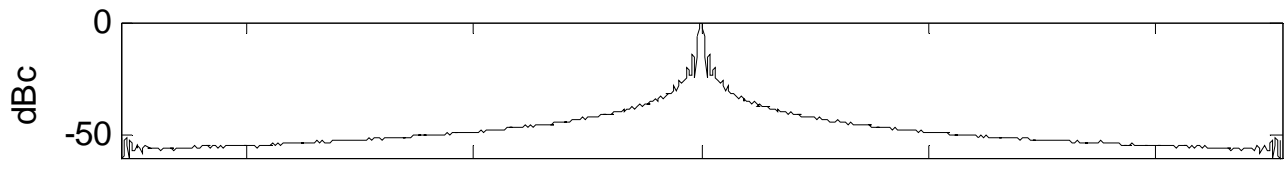

Figure 9. Principal axes cuts of the IPR. 


\subsection{Trapezoidal Aperture}

Sandia designed SAR systems collect and process raw data corresponding to a trapezoidal aperture. This accounts for the forked azimuth sidelobes in the IPR for finer resolutions. The trapezoidal aperture results from the real-time motion compensation employed, that varies waveform parameters as a function of collection geometry. ${ }^{10}$ The angle between the azimuth 'whiskers' is equal to the angle subtended during data collection.

Note that the azimuth IPR cuts the two-dimensional IPR between its whiskers.
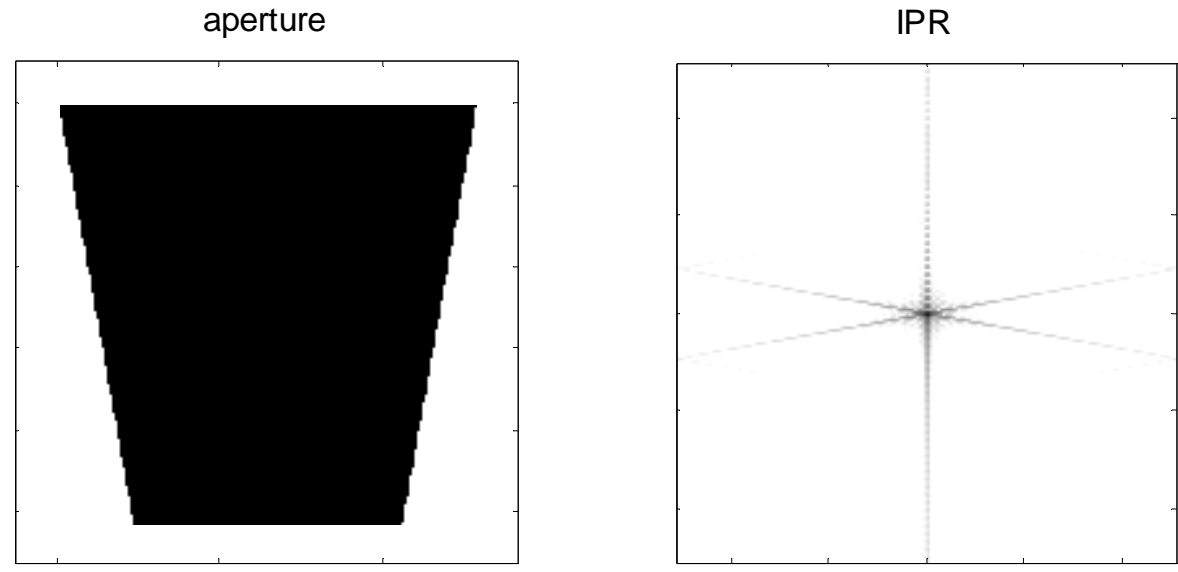

Figure 10. Trapezoidal aperture and corresponding IPR. The trapezoidal aperture corresponds to $0.0 .025 \mathrm{~m}$ resolution.

range IPR

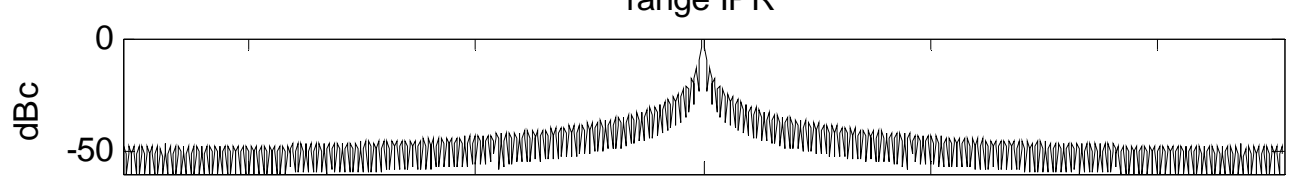

azimuth IPR

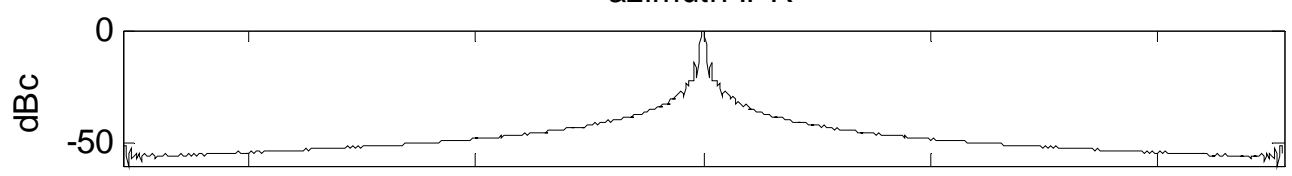

Figure 11. Principal axes cuts of the IPR. 


\section{Sidelobe Control of the IPR}

All finite area apertures will exhibit sidelobes. There is no escaping this. These are generally undesirable, in that they represent 'spilled' energy that may mask weaker nearby target points. Since sidelobes are a function of edges, they may nevertheless be reduced by 'softening' the edges of the aperture. This is effectively filtering the IPR for the purpose of sidelobe reduction, and is accomplished by applying 'window' functions to the raw Fourier-space data that taper the aperture. A side effect of this is that the mainlobe of the IPR is broadened by some amount, and the Signal-to-Noise Ratio (SNR) gain of the filter is generally reduced a small amount. In fact, this actually causes a slightly less accurate image rendering of the target scene, in the mean-squared error sense. Nevertheless, this is generally considered to be a good trade.

Window functions are one-dimensional functions that generally get applied in orthogonal directions to a two dimensional aperture. More specifically, they are normally applied just before performing one-dimensional Fast Fourier Transform (FFT) operations in corresponding dimensions.

Window functions and their characteristics abound in the literature. Sandia normally prefers the Taylor window function for SAR images (with parameters of $-35 \mathrm{~dB}$ sidelobe limit, and $\bar{n}=4$ ). Characteristics of this window function are given in the following figures. Subsequent sections of this report will presume this window function is employed, unless otherwise noted.

$-35 \mathrm{~dB}$ Taylor window taper function

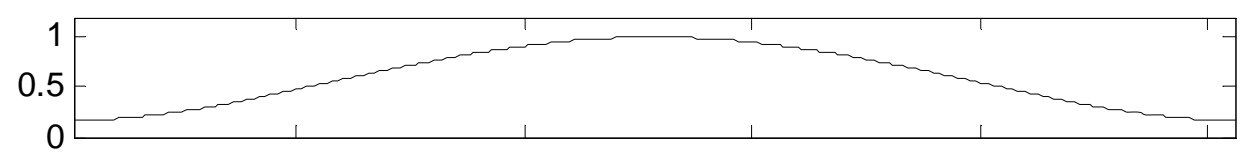

IPR
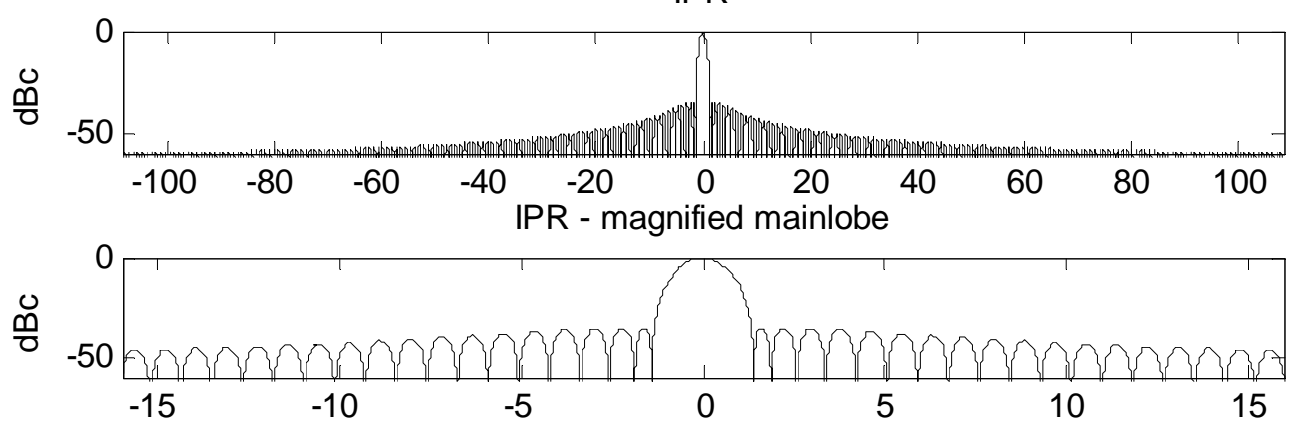

Figure 12. Taylor window characteristics (with parameters of $-35 \mathrm{~dB}$ sidelobe limit, and $\bar{n}=4$ ). The top plot is the taper function itself normalized to its peak value. The bottom plots are the one-dimensional IPR for this taper, with abscissa units equal to the $-3 \mathrm{~dB}$ width of the mainlobe. 


\subsection{Rectangular Aperture - Windowed}

For a rectangular aperture, the window function is applied in orthogonal directions, i.e. in range and azimuth directions. The IPR still has sidelobes, but they are at a lower level, and fall off earlier. Compare this to Figure 6.
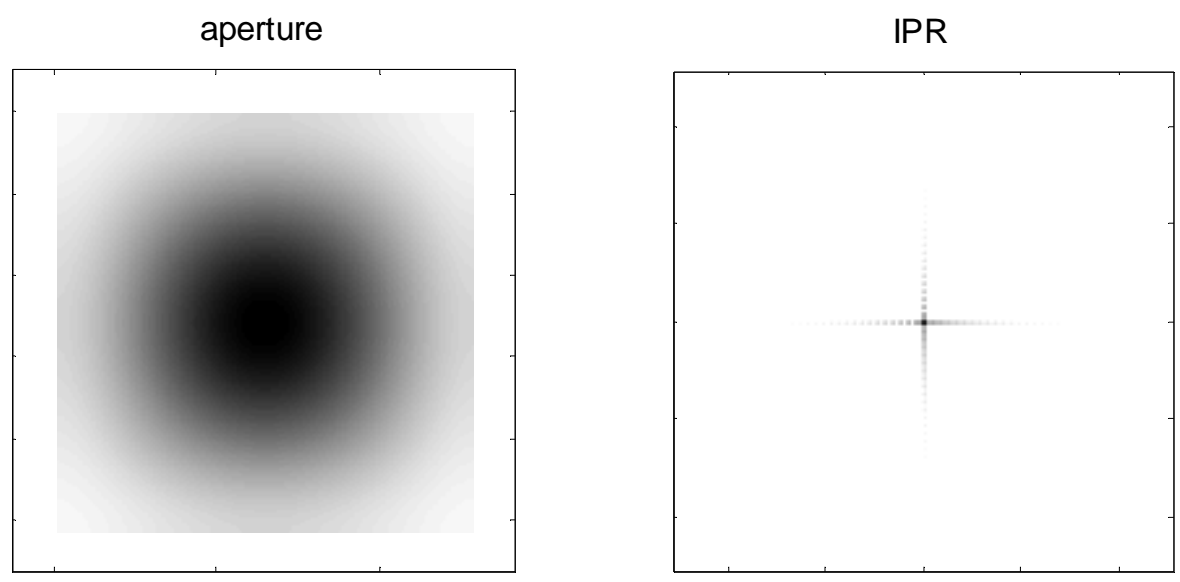

Figure 13. Rectangular aperture and corresponding IPR.

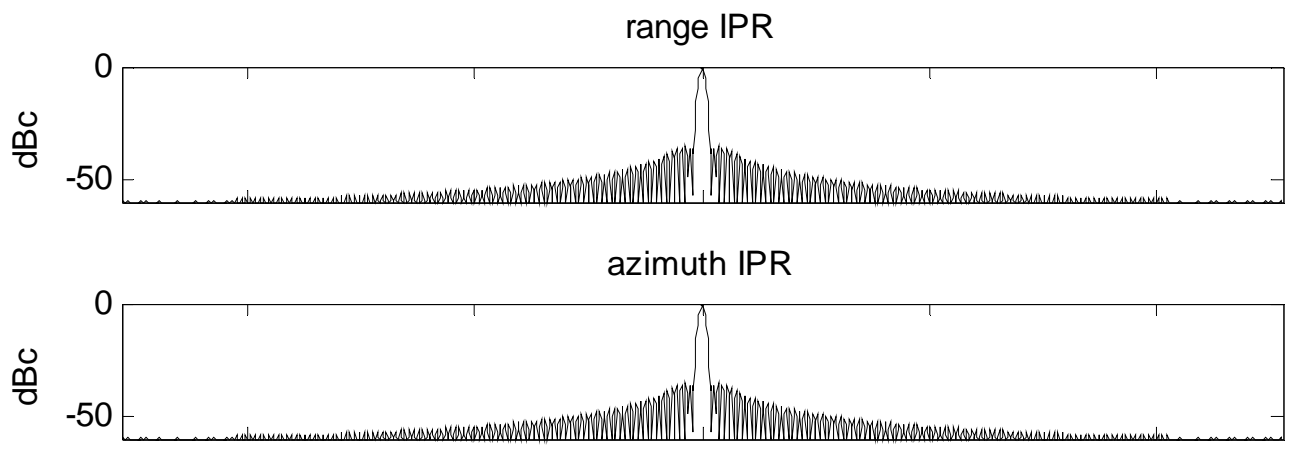

Figure 14. Principal axes cuts of the IPR. 


\subsection{Annular Ring Segment Aperture - Windowed}

For an annular ring segment aperture, the window function is applied in orthogonal directions, but differently than for the rectangular aperture. With respect to the origin, or center of the annulus, the left and right edges represent radial line segments, and top and bottom edges represent segments of circular arcs. The window function is applied in the radial direction, and along the circular arcs, but only over the extent of the data. The IPR still has sidelobes, but they are at a lower level, and fall off earlier. Compare this to Figure 8.
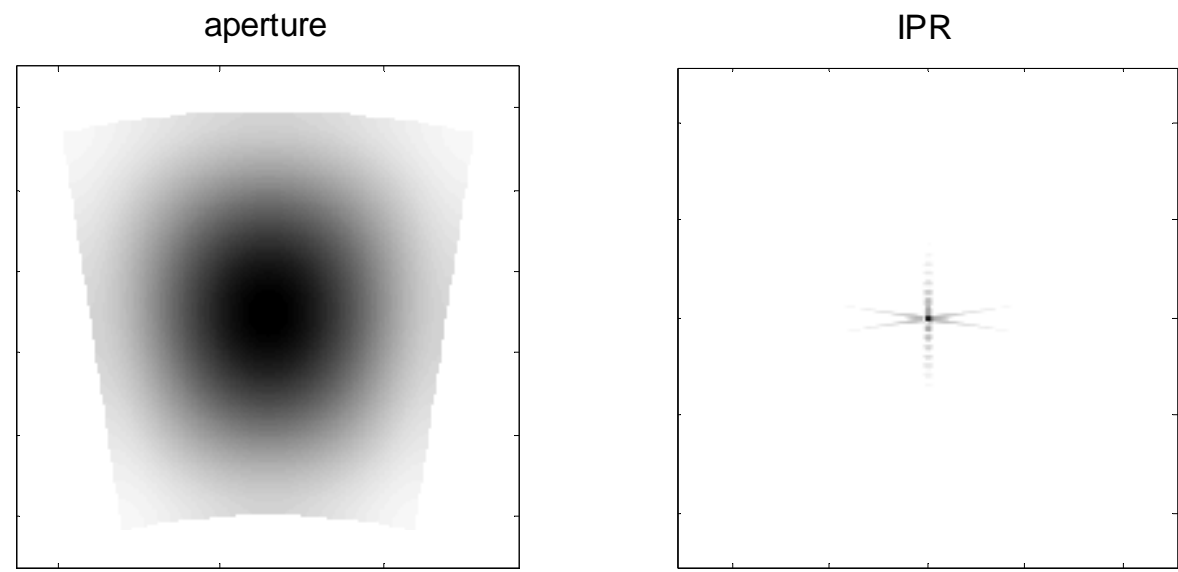

Figure 15. Annular ring segment aperture and corresponding IPR. The annular ring segment aperture corresponds to $0.025 \mathrm{~m}$ resolution
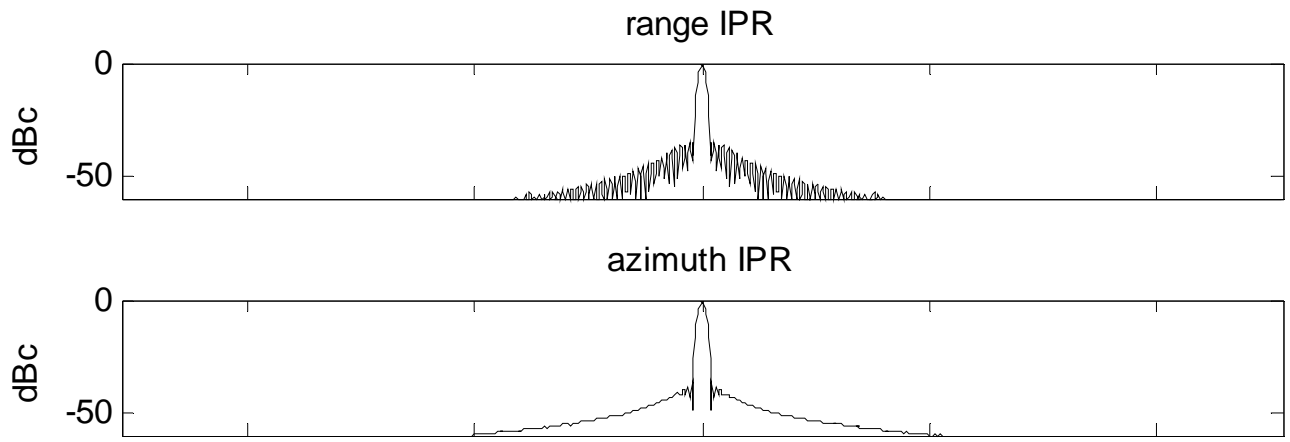

Figure 16. Principal axes cuts of the IPR. 


\subsection{Trapezoidal Aperture - Windowed}

For a trapezoidal aperture, the window function is applied effectively in orthogonal directions, nominal azimuth and range directions. As with the other examples, the IPR still has sidelobes, but they are also at a lower level, and fall off earlier. Compare this to Figure 10. This IPR is consistent with Sandia designed SAR systems, which process a trapezoidal aperture but with Taylor window functions for aperture weighting.
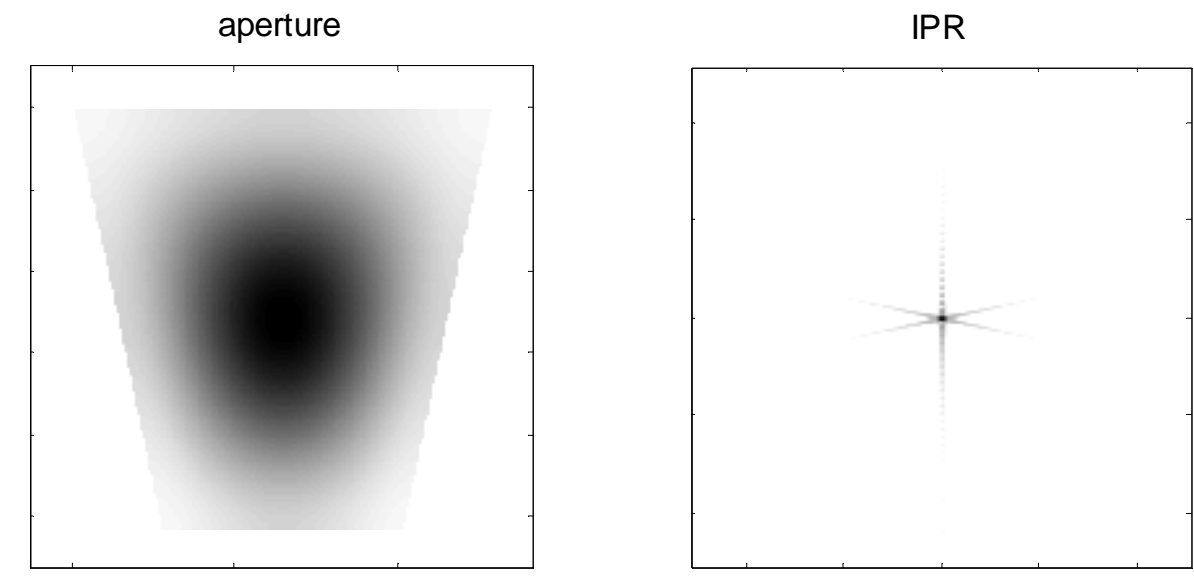

Figure 17. Trapezoidal aperture and corresponding IPR. The trapezoidal aperture corresponds to $\mathbf{0 . 0 . 0 2 5} \mathrm{m}$ resolution.
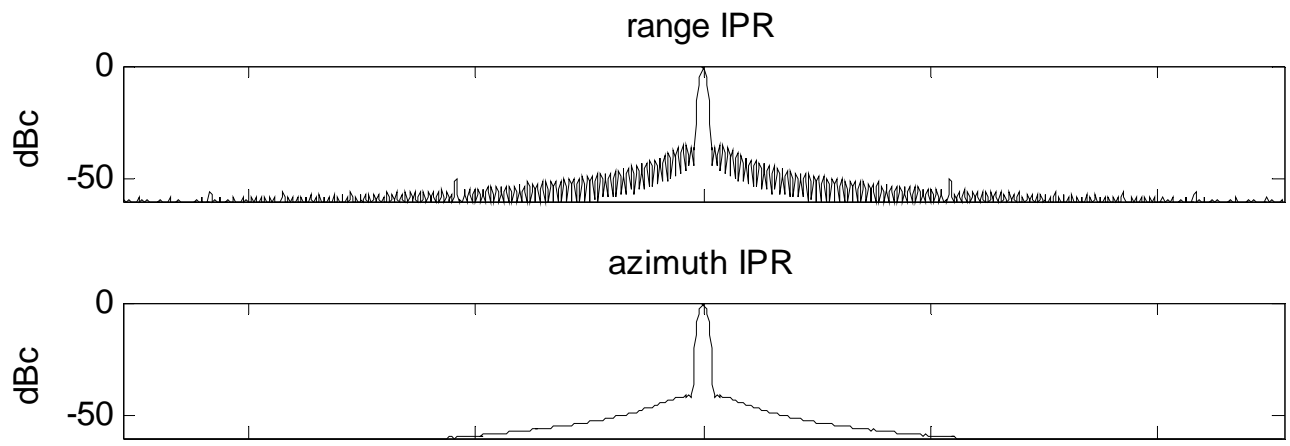

Figure 18. Principal axes cuts of the IPR. 


\section{IPR Specifications}

A typical IPR specification for a SAR system embodies the following elements

1) The $-3 \mathrm{~dB}$ width of the IPR mainlobe typically needs to correspond to the specified resolution, within some tolerance, often $10 \%$. This is generally taken to mean that the actual achieved resolution can not be degraded more than $10 \%$ from the nominal value. Resolution is specified in units of distance, such as perhaps meters, or inches, etc.

2) Peak sidelobes need to fall below some maximum specification. Often this is specified as an envelope curve, such as

$$
E n v_{d B}=20 \log _{10}\left(\left(\frac{u}{2.59}\right)^{-2}+1\right)-30 \quad \text { for }|u| \geq 1.5
$$

where $u$ is in units of the corresponding resolution of the radar, after the window is applied.

3) Integrated sidelobe energy needs to fall below a limit specified by the allowable Multiplicative Noise Ratio (MNR). Sidelobes, especially degraded sidelobes, are the principal source of multiplicative noise.

4) Sometimes, an IPR mainlobe width is also specified at some other level with respect to the peak of the mainlobe. For example, the $-18 \mathrm{~dB}$ width of the IPR mainlobe might be specified. Note that equation (1) specifies the $-18 \mathrm{~dB}$ width at 3 resolution unit widths.

5) Pixel spacing is usually specified as some fraction of the resolution. Often this is via an oversampling factor, defined as the ratio of nominal resolution to pixel spacing. Practical signal processing concerns dictate that this must be greater than or equal to the broadening factor of the window function, sometimes called the window bandwidth. For the $-35 \mathrm{~dB}$ Taylor window, this is 1.1822.

6) The absolute brightness or peak value of the IPR is generally specified via an RCS calibration specification. For example, the RCS indication of the image might need to be within $10 \mathrm{~dB}$ of the actual value.

A radar designer will use these specifications to select an appropriate window function for signal processing, which sets the basic shape of the IPR, especially the region near its mainlobe response. Of these, items 1-4 specify aspects of the shape of the IPR. Item 5 is a function of zero padding prior to the FFT. Item 6 refers to gain calibrations. 
We also mention that IPR specifications may at times be placed on individual components, such as the Transmitter Power Amplifier as a means of specifying their linearity characteristics. ${ }^{11}$ These are often stricter than the system specification. Nevertheless, we will restrict subsequent discussion to system IPR specifications.

\subsection{The Taylor Window}

The sample requirements of the previous section are easily met by the Taylor window. Specifically, the $-35 \mathrm{~dB}$ Taylor window with parameter $\bar{n}=4$, is favored by Sandia for SAR images. Figure 19 illustrates this particular Taylor window function's IPR with respect to the IPR specifications given in the previous section. A well focused SAR image should achieve a nearly perfect IPR, with principal axis cuts nearly identical to those shown, down to the clutter or noise floor.
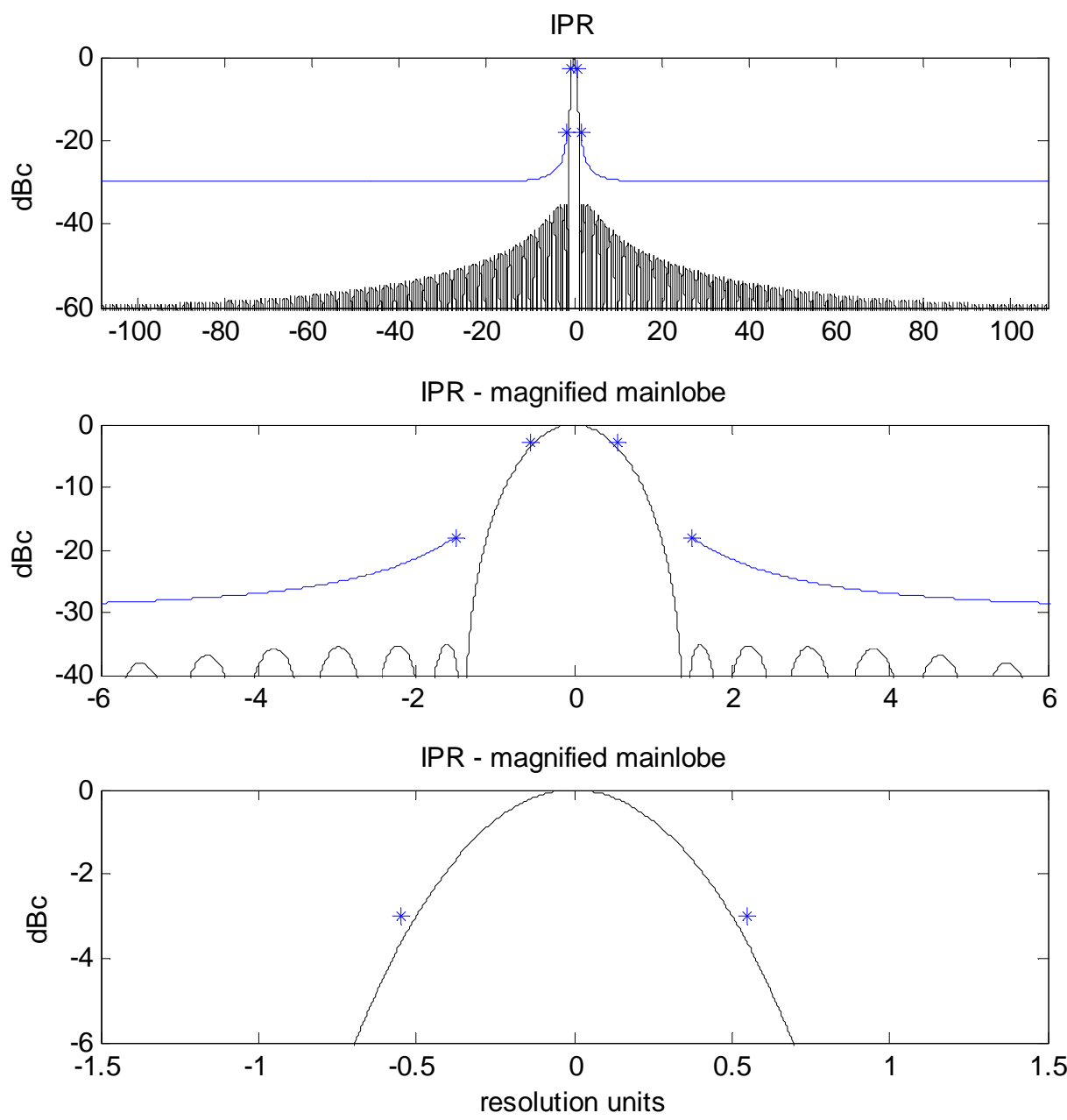

Figure 19. Taylor window IPR related to IPR specifications at various magnifications. Abscissa axis units are equal to the $-3 \mathrm{~dB}$ resolution. 
We further identify the following characteristics of this Taylor window.

Table 1. Properties of $-35 \mathrm{~dB}$ Taylor window with $\bar{n}=4$.

\begin{tabular}{|l|c|}
\hline Window IPR $-3 \mathrm{~dB}$ bandwidth (broadening factor) & 1.1822 \\
\hline Window IPR $-18 \mathrm{~dB}$ bandwidth relative to $-3 \mathrm{~dB}$ bandwidth & 2.2087 \\
\hline Window SNR gain with respect to uniform weighting & $-0.914 \mathrm{~dB}$ \\
\hline Window peak sidelobe level & $-35.1 \mathrm{~dB}$ \\
\hline Window integrated sidelobe level (outside of first nulls) & $-27.1 \mathrm{~dB}$ \\
\hline
\end{tabular}

\subsection{Relationship of Pixel Spacing to Resolution}

Resolution, like other equivocal terms such as bandwidth, may be defined with any of a number of different criteria, any of which is preferred in unique circumstances.

Nevertheless, a common definition is the width of the IPR at points $-3 \mathrm{~dB}$ with respect to the peak of the IPR. We adopt this convention unless otherwise noted.

We illustrate some points about resolution and pixel spacing with the following analogous development.

Consider a signal sampled at some frequency $f_{s}$, with $N_{\text {data }}$ uniformly spaced samples. The total time span of the samples is

$$
T=N_{\text {data }}\left(\frac{1}{f_{s}}\right) .
$$

The nominal frequency resolution of this data is often given by

$$
\rho_{f, \text { nominal }}=\frac{1}{T}=\frac{f_{S}}{N_{\text {data }}} .
$$

However, if an FFT were taken of this data, with no weighting or window function (i.e. uniform weighting), then the $-3 \mathrm{~dB}$ width of the IPR would be measured at

$$
\rho_{f, \text { uniform },-3 \mathrm{~dB}}=(0.8845) \frac{1}{T}=(0.8845) \rho_{f, \text { nominal }} \cdot
$$


If the $-35 \mathrm{~dB}$ Taylor window of the previous section were applied before the FFT, then the $-3 \mathrm{~dB}$ width of the IPR would be measured at

$$
\rho_{f, \text { Taylor, }-3 \mathrm{~dB}}=(1.1822) \frac{1}{T}=(1.1822) \rho_{f, \text { nominal }} .
$$

This factor of 1.1822 is the IPR broadening factor of Table 1 . This is a constant that permeates calculations of other radar parameters. For example, both radar bandwidth and synthetic aperture length are extended by this factor to compensate for the otherwise broadening of the IPR by this amount. We define this constant for whatever window function we intend to use as

$$
a_{w}=\text { IPR broadening factor. }
$$

Now suppose that after application of the window function we append $N_{\text {zeros }}$ empty (or zero) samples to the data. The FFT length is then

$$
N_{F F T}=N_{\text {data }}+N_{\text {zeros }} .
$$

The nominal frequency resolution with uniform weighting of this data remains

$$
\rho_{f, \text { nominal }}=\frac{f_{s}}{N_{\text {data }}}
$$

and the $-3 \mathrm{~dB}$ width of the IPR after using the Taylor window function remains

$$
\rho_{f, \text { Taylor, }-3}=a_{w} \frac{f_{s}}{N_{\text {data }}} .
$$

However, the FFT output vector frequency spacing (pixel spacing in an image) becomes

$$
\delta_{f}=\frac{f_{s}}{N_{F F T}}
$$

regardless of what window function was used or not used. Since $N_{F F T} \geq N_{\text {data }}$, we are constrained with

$$
\delta_{f} \leq \frac{1}{a_{w}} \rho_{f}
$$

that is, the FFT output frequency spacing must be less than the resolution by at least the factor $1 / a_{w}$. Specifically, let us define the oversampling factor 


$$
a_{o s}=\frac{\rho_{f}}{\delta_{f}} .
$$

Then we can calculate

$$
N_{\text {data }}=N_{F F T} \frac{a_{w}}{a_{o s}}
$$

and realize the constraint

$$
a_{o s} \geq a_{w}
$$

From this analysis, we realize the following points.

- Resolution depends on the extent of the data, in our example the total time span $T$. For SAR this is analogous to the diameter of the two-dimensional aperture, that is, the microwave RF bandwidth for range resolution, and the angular extent of the data collection for azimuth resolution.

- $\quad$ Larger apertures yield finer resolutions.

- Window functions applied before FFTs will broaden the IPR in the direction of window function application. This is by a predictable amount for the window function employed.

- Both microwave RF bandwidth and the angular extent of the data collection can be extended during data collection so that after the window functions are employed and FFTs performed, the IPR width is 'broadened' to the desired width. That is, we collect extra data so that the IPR broadening due to window functions is precisely compensated.

- Image pixel spacing is limited to be finer than the resolution in the corresponding dimension.

- Image pixel spacing, subject to some maximum value, can be effectively arbitrarily specified by suitable zero-padding of the windowed data prior to FFT application. Similar results can be obtained by using the Chirped Z-Transform $(\mathrm{CZT}){ }^{12}$

- Note also that if the abscissa units were not Taylor window resolution units, but rather in units of $f_{s} / N_{\text {data }}$, then the $-3 \mathrm{~dB}$ width of the Taylor window IPR mainlobe would measure to 1.1822, and a 10\% broadening limit beyond this would place the boundary at 1.3 of these new units. 
Give me six hours to chop down a tree and I will spend the first four sharpening the axe.

-- Abraham Lincoln 


\section{Effects of Non-Ideal Radar Performance on IPR}

If the IPR is a measure of the SAR's goodness, then a suboptimal IPR is an indication of the SAR's suboptimal performance. Usually the first indication of a hardware or software anomaly in a SAR system is an anomaly in the IPR itself. Consequently, one of the best troubleshooting tools to the SAR engineer is a careful analysis of the IPR.

In this section we address non-ideal SAR performance on the IPR. Specifically, we will illustrate various anomalies in a one dimensional IPR. Except in some special cases, it is convenient to investigate phase errors separately from amplitude errors as applied to the one-dimensional aperture prior to Fourier transformation.

We will then give examples of what might cause such errors in the azimuth IPR, and what might cause such errors in the range IPR.

We note that azimuth phase errors are usually correctable by an autofocus operation. Simple autofocus addresses a uniform (affecting all points in the image equally) phase error function. More elaborate autofocus operations address spatially variant phase errors.

Uniform (affecting all points in the image equally) range phase errors are usually correctable with Phase-Error-Correction (PEC) or predistortion of the LFM chirp phase that is generated by the Digital Waveform Synthesizer (DWS). ${ }^{13}$

\section{Delay Line}

When using a delay line for an IPR measurement, special care should be taken to ensure that the characteristics of the delay line are accommodated in the test. For example, the delay line (if distortionless) offers only a constant delay, corresponding to a constant range. Any motion dependent waveform control needs to account for the fact that the delay line doesn’t offer motion dependent range behavior.

Furthermore, the delay line is ideally a distortionless channel. In practice it is actually not, and offers its own frequency dependent gain and phase behavior. This needs to be accounted for in any range IPR analysis. For example any PEC calculations that involve the delay line needs to subtract the effects of the delay line to be accurate for the radar system by itself. 


\subsection{Quadratic Phase Errors}

The most obvious effect of a quadratic phase error is to symmetrically broaden the IPR mainlobe. This is illustrated in Figure 20 for 90 degrees of peak quadratic phase error. Note that this amount places the IPR mainlobe right at the allowable $-3 \mathrm{~dB}$ and $-18 \mathrm{~dB}$ widths. Larger peak quadratic phase errors cause more broadening.

A uniform azimuth IPR with this characteristic usually indicates motion measurement errors, either a constant along-track velocity error, or a line-of-sight acceleration error.

A range IPR with this characteristic indicates an uncompensated dispersion, probably due to a microwave RF filter. It might also be caused by inadequate correction for Doppler frequency scaling due to high line-of-sight velocity.
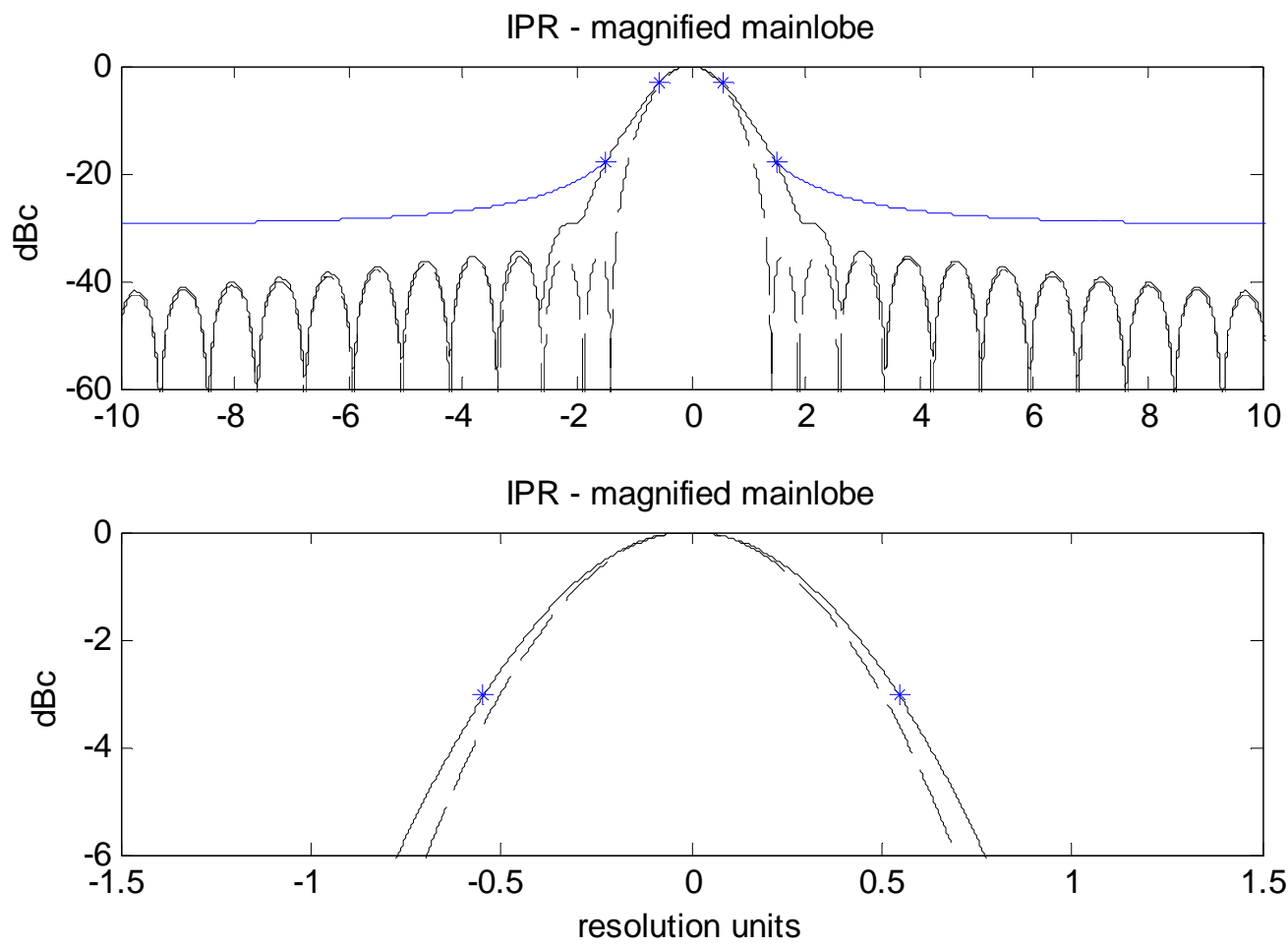

Figure 20. IPR with 90 degrees of quadratic phase error. 


\subsection{Cubic Phase Errors}

The most obvious effect of a cubic phase error is to asymmetrically broaden the IPR mainlobe and to raise sidelobes on one side of the mainlobe. This is illustrated in Figure 21 for 90 degrees of peak cubic phase error. Note that this amount causes the IPR's innermost raised sidelobe to just exceed the envelope limit. Larger peak cubic phase errors cause more broadening and higher sidelobes. A purely cubic term will also shift the mainlobe location. Figure 21 has been corrected for this.

A uniform azimuth IPR with this characteristic usually also indicates motion measurement errors.

A range IPR with this characteristic likely indicates an uncompensated microwave RF filter. It may also indicate inadequate return-loss in a section of cable that is on the order of a resolution unit long. Inadequate return loss may be caused by loose connectors, damaged cables, faulty construction, etc.
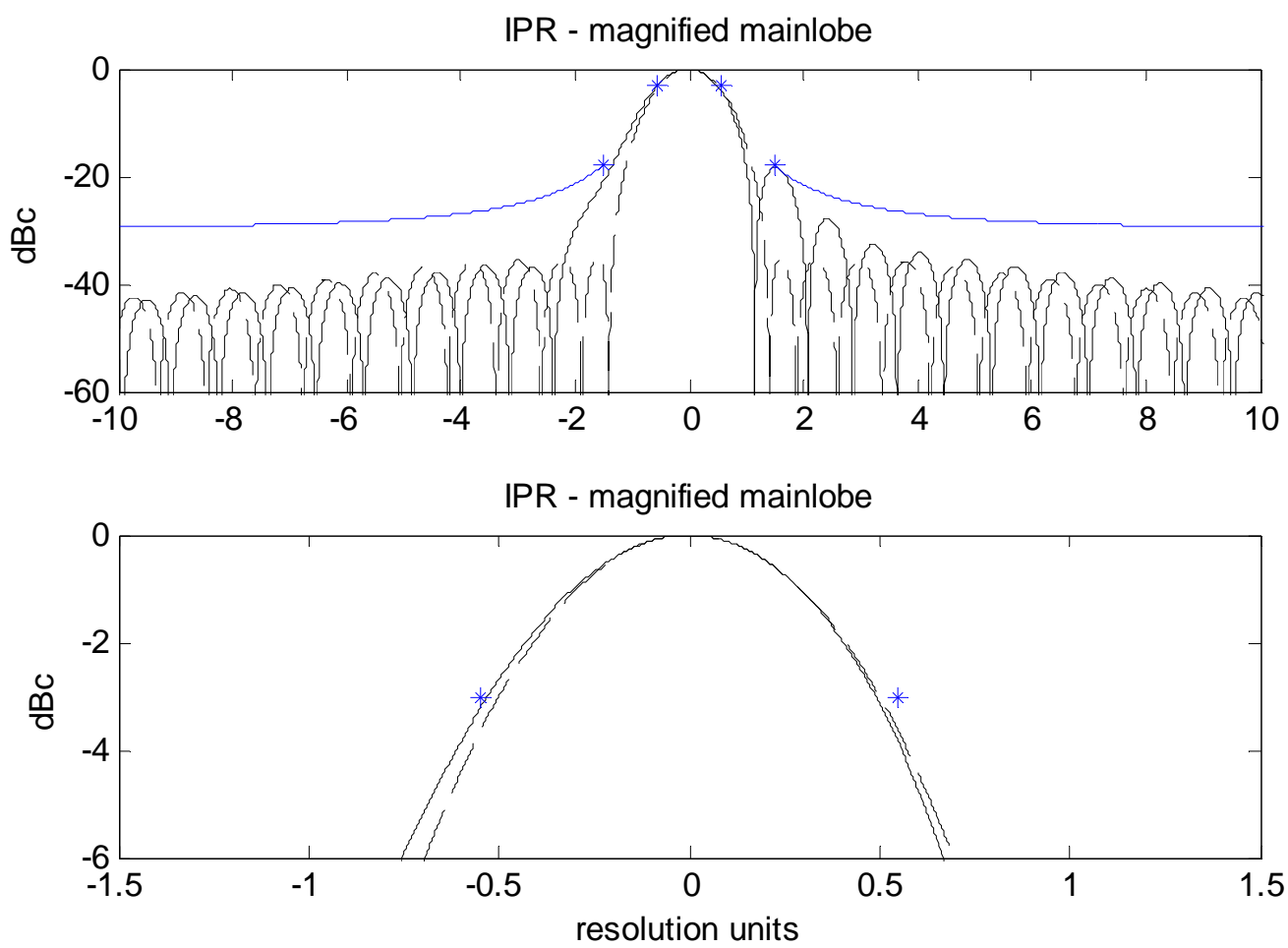

Figure 21. IPR with 90 degrees of cubic phase error. 


\subsection{Sinusoidal Phase Errors}

Sinusoidal phase errors are a phase ripple or phase modulation to the radar signal. As such, they cause symmetric raised sidelobes in the IPR. SAR systems are exceptionally sensitive to sinusoidal phase errors because the raised sidelobes may occur some distance from the mainlobe, appearing as ghost targets in the direction of the sinusoidal errors. This is illustrated in Figure 22 for 3 degrees of sinusoidal phase ripple with 7 cycles across the aperture.

A uniform azimuth sinusoidal phase error is most likely due to a vibration of the antenna, perhaps due to an unstable control loop. Other vibrating components may sometimes cause similar symptoms. Multipath reflections from an aircraft propeller may also manifest themselves as an azimuth sinusoidal phase modulation.

A uniform range sinusoidal phase error suggests a passband phase ripple where the radar waveform is still a LFM chirp. Most often this is the transmitter power amplifier. Other amplifiers can also exhibit passband phase ripple. For many microwave RF applications passband phase ripple is tolerable, but not for SAR.
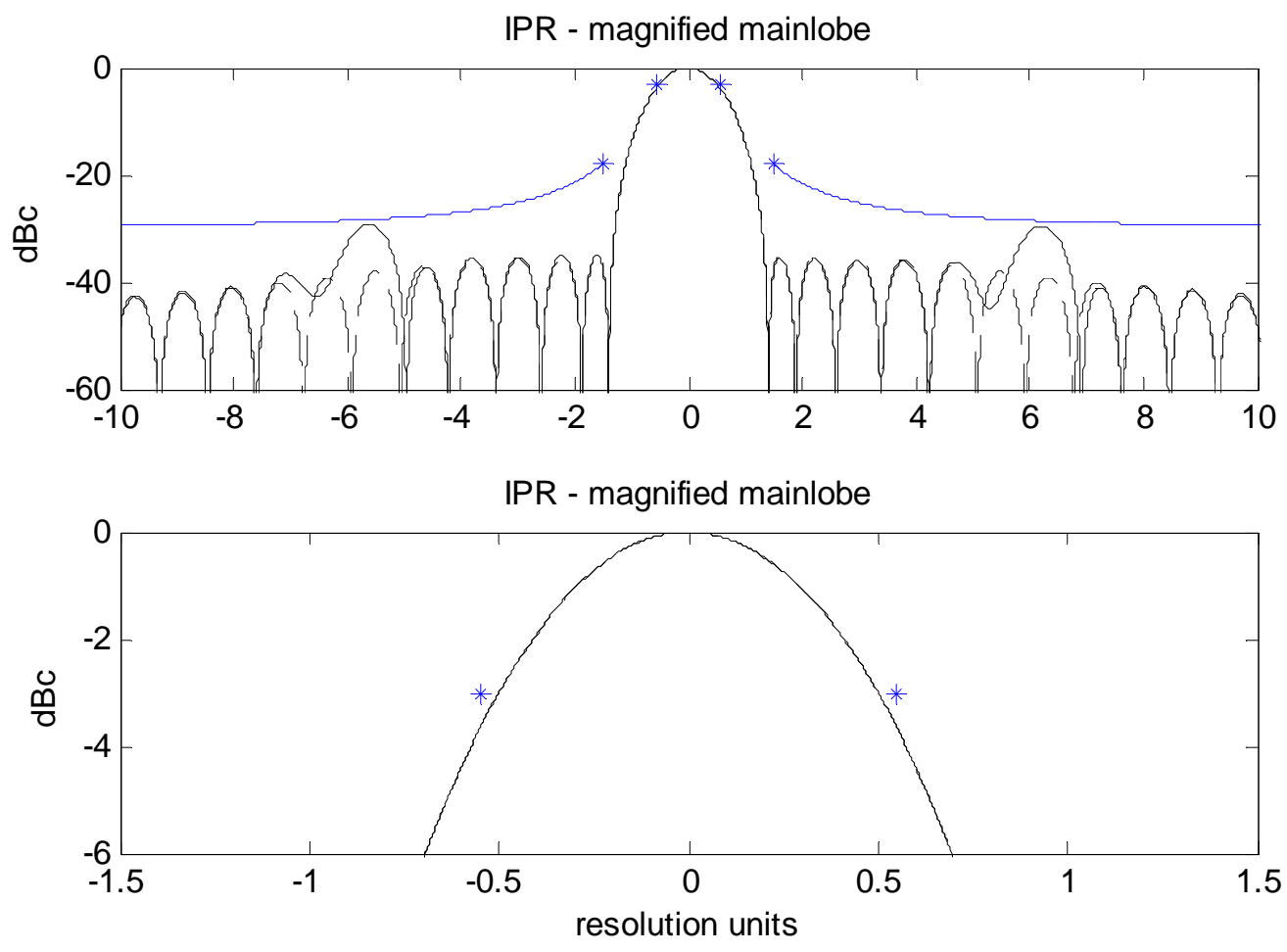

Figure 22. IPR with 3 degrees of sinusoidal phase error. 


\subsection{Amplitude Taper}

Low frequency amplitude fluctuations such as amplitude tapers will manifest themselves mainly in altering the mainlobe characteristics of the IPR rather than the far out sidelobe structure. For example, window functions themselves may be considered as effectively a single cycle of amplitude fluctuation or ripple. A taper is an even lower frequency of amplitude modulation than a window function. Figure 23 shows the effects of a linear gain variation of $10 \mathrm{~dB}$ from one end of the aperture to the other. Note that the effect is rather small.

In azimuth, a low frequency gain slope might be caused by atmospheric phenomena. If an antenna is not slewed in a spotlight fashion, then low frequency gain variations might be induced in the data due to antenna pattern effects.

In range, such a gain slope might be caused most likely by the transmitter power amplifier, although other amplifiers might also exhibit this characteristic. ${ }^{11}$
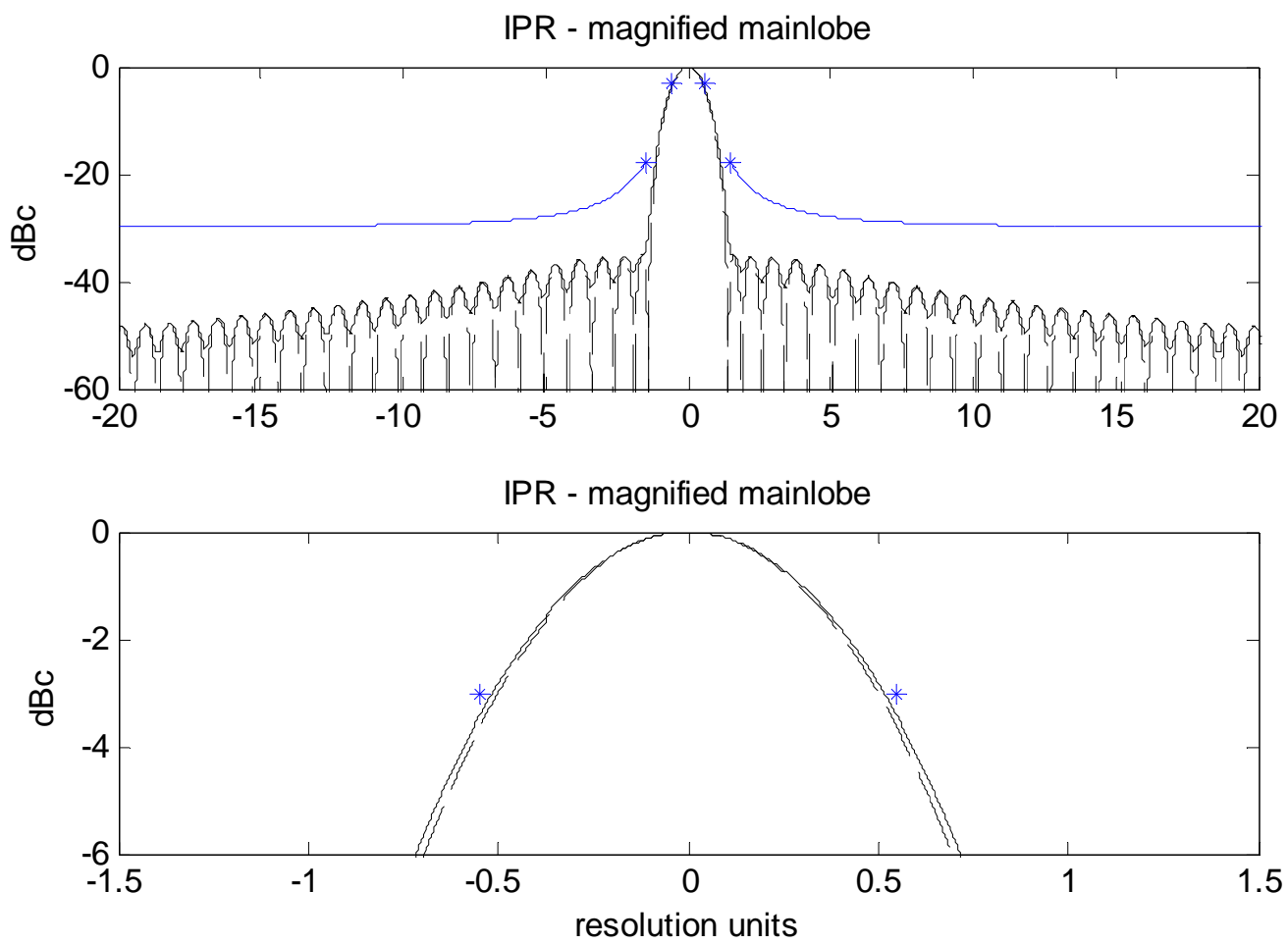

Figure 23. IPR with $-10 \mathrm{~dB}$ of linear gain variation. 


\subsection{Amplitude Ripple}

Sinusoidal amplitude errors are a ripple or modulation of the amplitude of the radar signal. As such, they cause symmetric raised sidelobes in the IPR much like phase ripple. SAR systems are also exceptionally sensitive to sinusoidal amplitude errors because the raised sidelobes may occur some distance from the mainlobe, appearing as ghost targets in the direction of the sinusoidal errors. This is illustrated in Figure 24 for $0.7 \mathrm{~dB}$ of sinusoidal amplitude ripple with 7 cycles across the aperture.

A uniform azimuth sinusoidal amplitude error is most likely due to a vibration of the antenna, perhaps due to an unstable control loop. Multipath reflections from an aircraft propeller may also manifest themselves as an azimuth sinusoidal amplitude modulation. Other multipath reflections may do so as well.

Analogous to phase ripple, a uniform range sinusoidal amplitude error suggests a passband amplitude ripple where the radar waveform is still a LFM chirp. Most often this is the transmitter power amplifier. Other amplifiers can also exhibit passband amplitude ripple. For many microwave RF applications passband amplitude ripple is tolerable, but not for SAR.
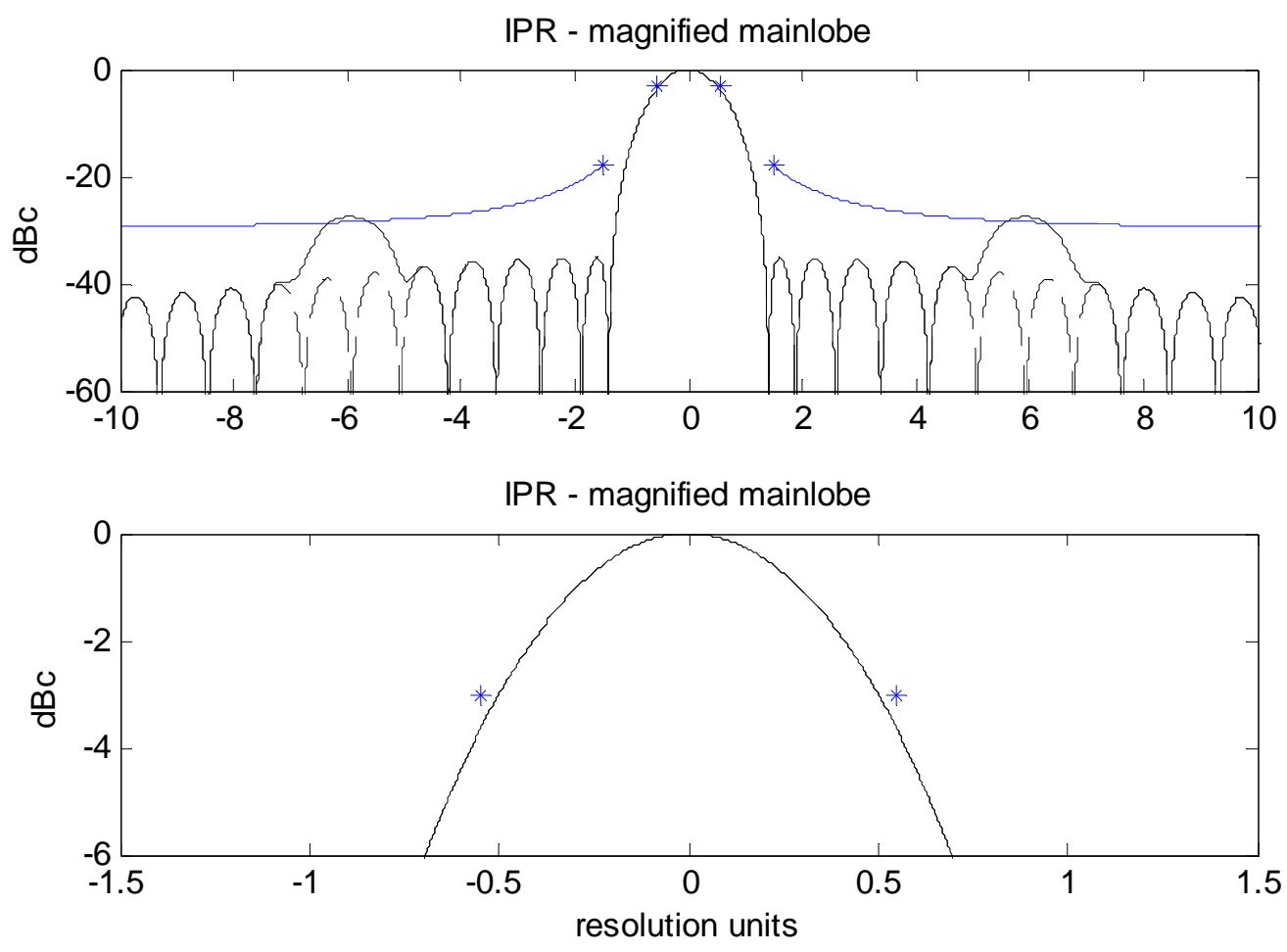

Figure 24. IPR with $0.7 \mathrm{~dB}$ of sinusoidal amplitude error. 


\subsection{Latent (Offset) Ghost Echoes}

Sinusoidal strictly phase errors, and strictly amplitude errors cause symmetric paired echoes. However, if a singular target encounters multiple simultaneous echo delays in an image, analogous to multipath, then these ghosts will appear as distinct targets some distance from the mainlobe but on only one side of the mainlobe. This is illustrated in Figure 25 with a secondary echo reduced by $30 \mathrm{~dB}$ from the mainlobe peak.

A uniform azimuth response of this nature is most likely due to a discontinuity in the focusing of the SAR. This can represent a malfunction of the autofocus operation, perhaps due to excessive residual range migration from inadequate motion measurement. ${ }^{14,15}$ Sufficient excessive range migration may cause multiple ghost echoes principally in azimuth, but may have a slight range offset, too.

A uniform range response of this nature is likely caused by poor transmission line return loss in the microwave RF circuitry, most probably the cables. This may be due to loose connectors, damaged cables, faulty construction, etc. The distance between the mainlobe and the secondary lobe is the electrical delay produced by the multiple reflections, and is proportional to the distance between impedance discontinuities.
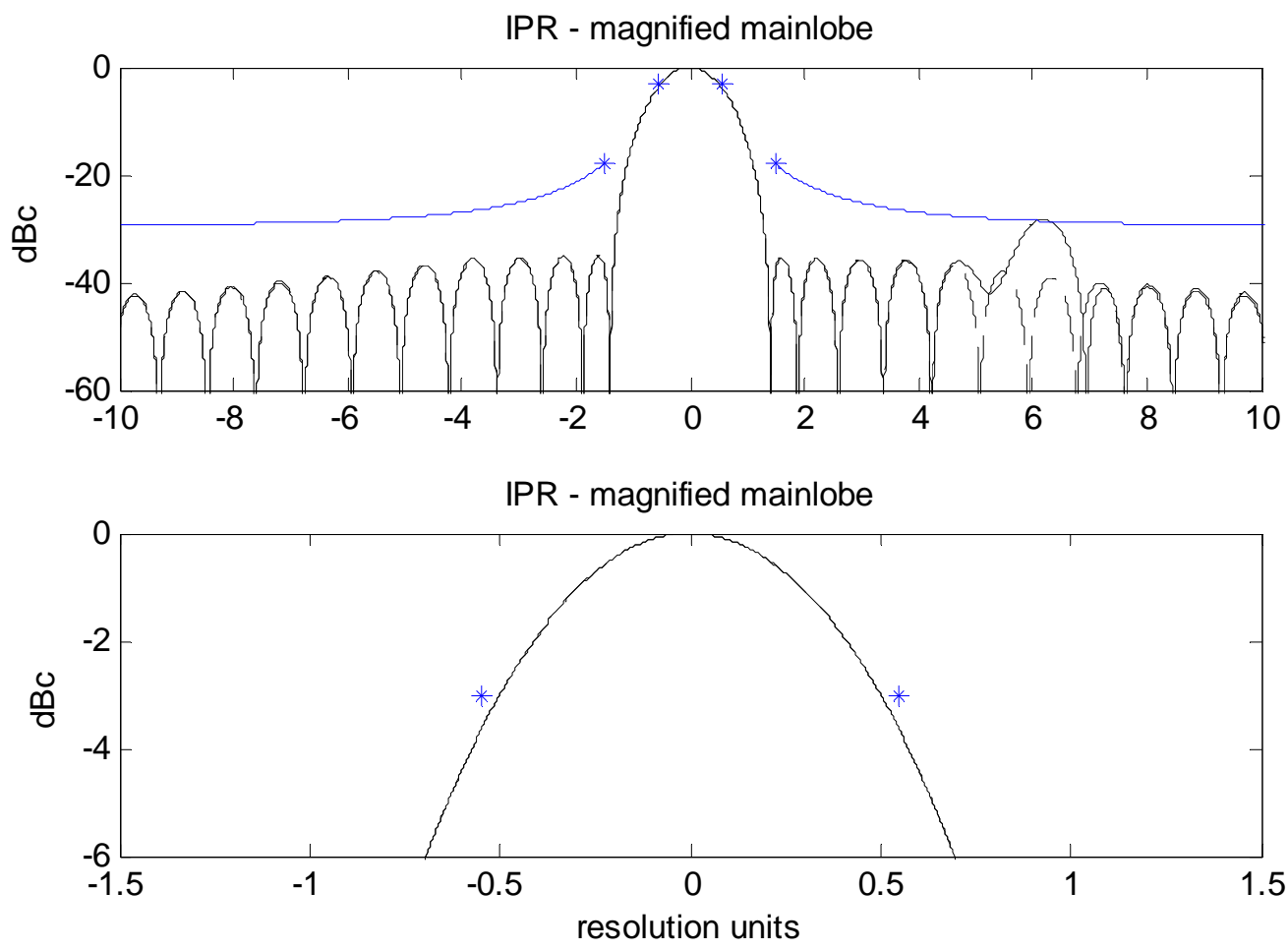

Figure 25. IPR with secondary ghost echo at $-30 \mathrm{dBc}$. 


\subsection{Spatially Variant Phase Errors}

Most SAR image formation algorithms are approximations to the matched filter required for each and every pixel in an image. This is the trade for efficient real-time algorithms. Consequently, the fidelity of image reconstruction from the raw data tends to diminish with distance from the focal point of the image, typically the scene center. That is, locations at the edges of an image are degraded in their IPR from those near the center of the image. This is usually manifested as a degraded azimuth IPR rather than a degraded range IPR. The radar designer accounts for this in his design by matching the image formation algorithm to the imaging geometry and image size required. As a result, this structural spatially variant IPR characteristic is typically not noticeable in the SAR image.

If a spatially variant IPR characteristic is noticeable in an image, such as is illustrated in Figure 26, then this may signal an along-track motion measurement error, i.e. improper PRF modulation as a function of inadequate velocity measurements. However, at certain imaging geometries, this might be more likely due to atmospheric effects beyond the control of the radar. ${ }^{16}$

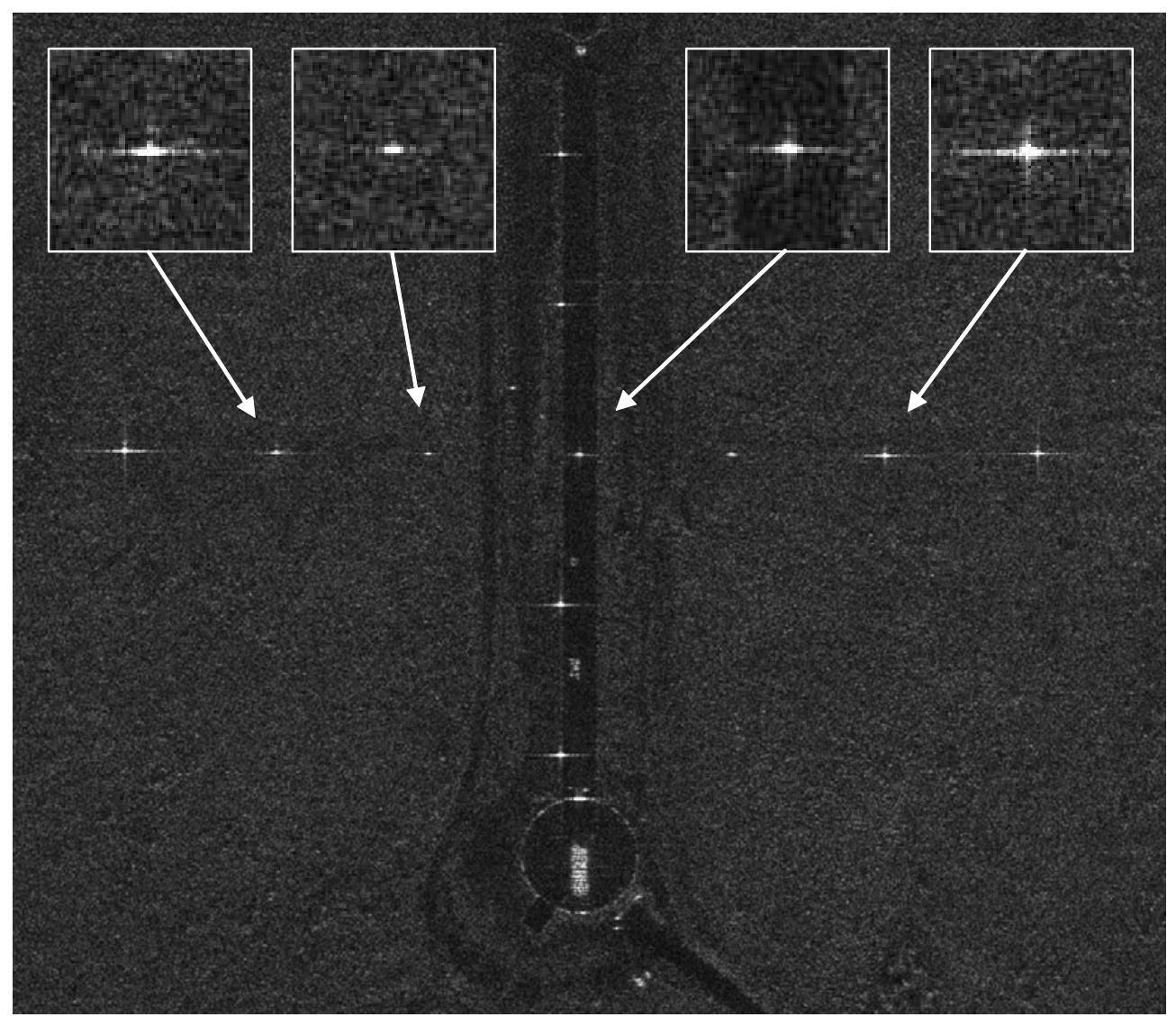

Figure 26. Spatially variant sidelobe structures signaling spatially variant phase errors. 


\subsection{Excessive Amplitude}

Modern high-performance SAR systems typically use floating-point calculations for all but highest-speed calculations such as presuming, etc. Nevertheless, there remain two principal choke points for dynamic range. The first, and most important, is the Analogto-Digital Converter (ADC). The second is the output detection operation that seeks to render an image in some finite number of bits, usually a small number to facilitate transmission in a fairly low bandwidth channel.

If an excessively large signal is put to the ADC, then the ADC operation becomes nonlinear. Optimal design is to limit or clip the signal. As nonlinear operations are wont to do, this adds harmonics to the original signal. Figure 27 illustrates the IPR of a complex sinusoid clipped at $90 \%$ of its peak value real and imaginary components. This may occur in either range or azimuth dimensions, or both dimensions. Causes for this might include improper gain calibration, or improper signal level selection for delay line measurements

If the clipping occurs after the FFT, then the IPR itself is clipped. This has the effect of lowering the envelope and other width measuring points.
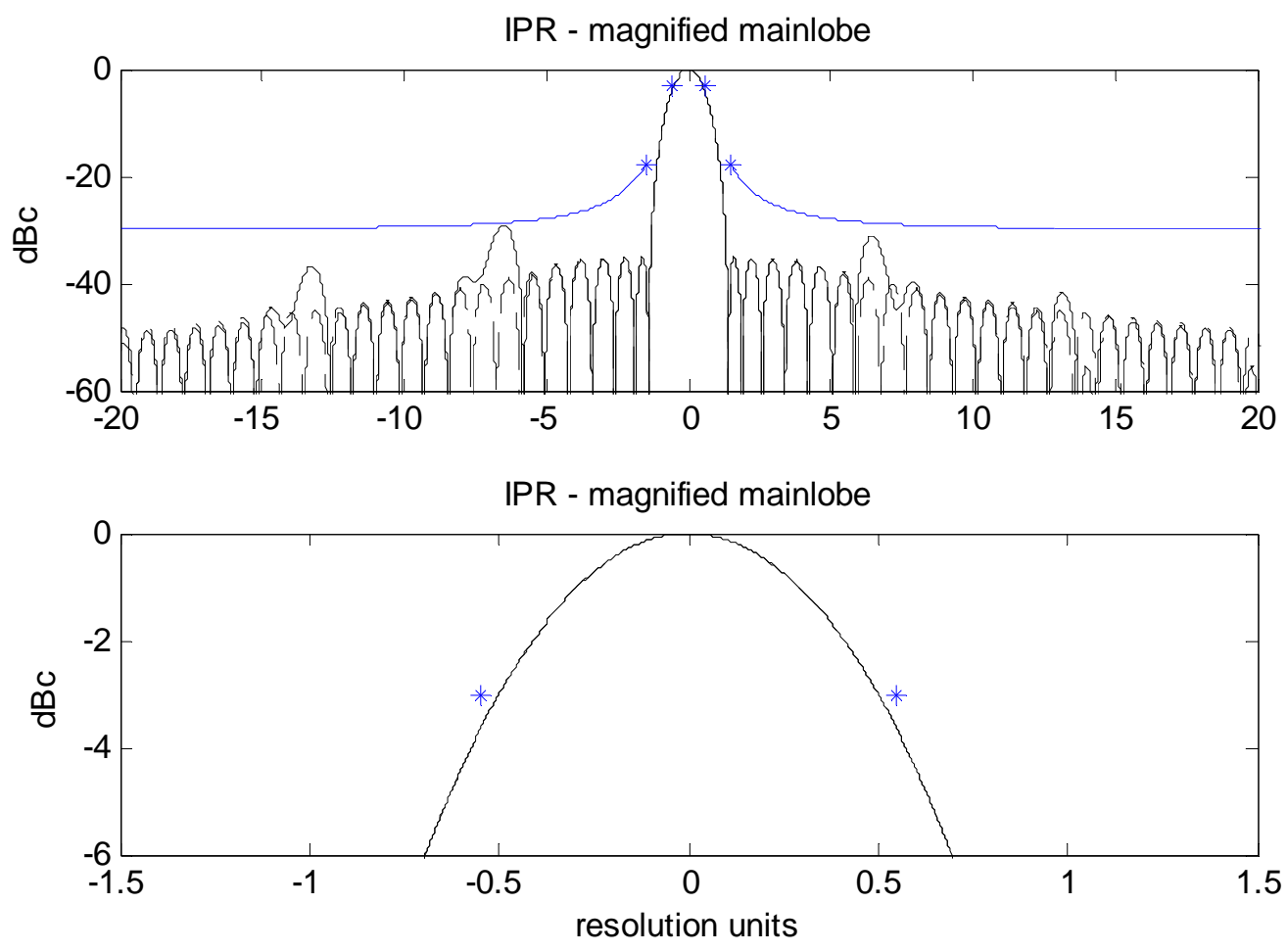

Figure 27. IPR with raw signal clipped at $90 \%$ level. 


\subsection{Quantization Noise}

An ADC naturally quantizes its input signal. This is fundamental to ADC operation. Small signals are quantized to relatively few levels. In the absence of mitigating techniques, the quantization noise is highly correlated with the small signal. The consequence is coherent anomalies that are a function of the input signal, most generally anomalous sidelobes as illustrated in Figure 28. The purpose of dithering, via ensuring adequate noise at the ADC input, is to whiten the quantization noise. This smears the quantization noise into a low-level background noise, often below the additive thermal noise floor of the image.

If quantization effects are visible as coherent anomalies rather than broadband background noise, this is an indication of inadequate dithering, perhaps due to inadequate gain with no additional dithering noise source.
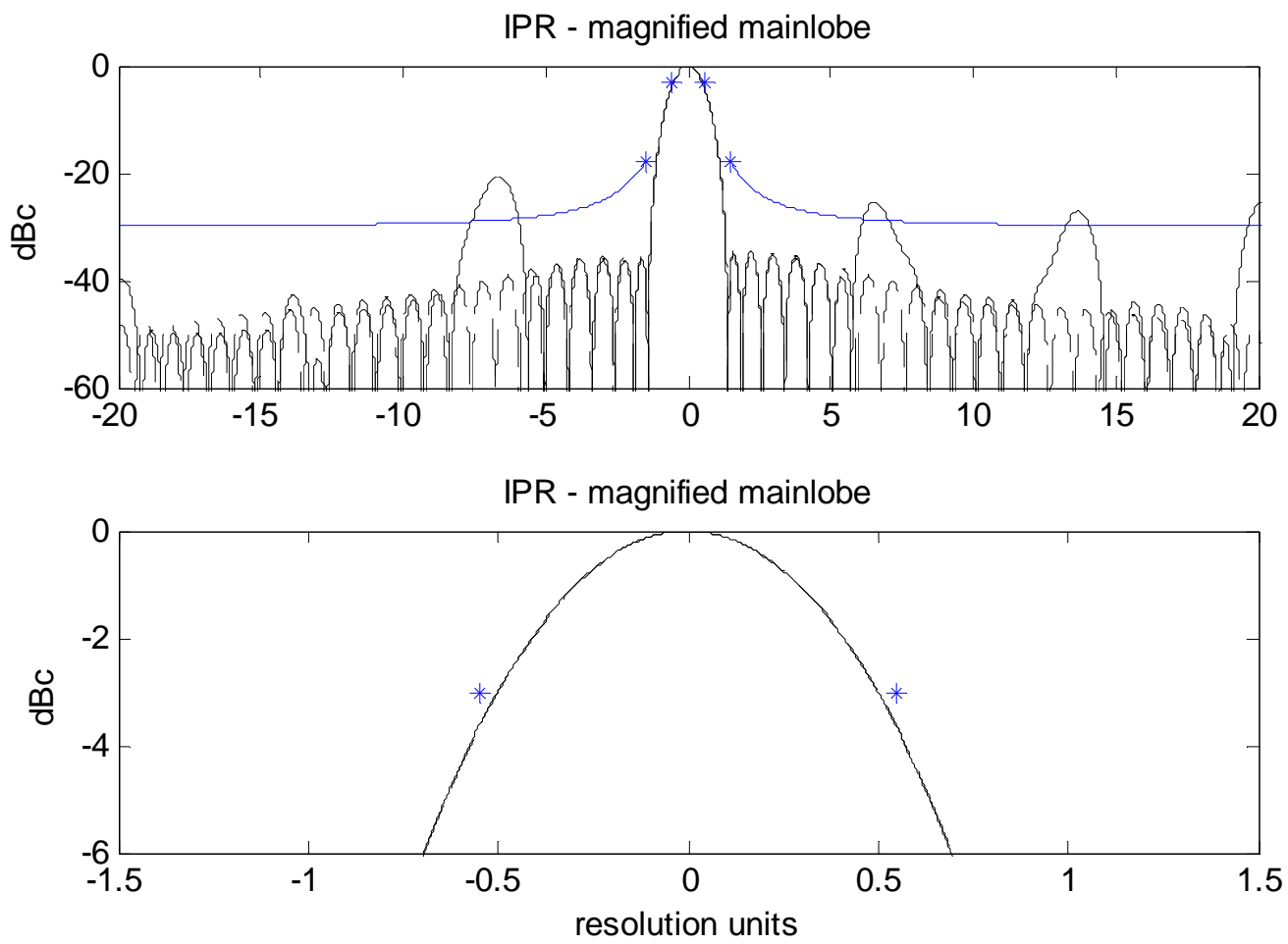

Figure 28. IPR with inadequate dithering noise source. 


\section{Summary Discussion and Conclusions}

The principal measure of the proper operation of radar hardware and software is the Impulse Response. The complete IPR is a two-dimensional entity, although substantial information exists in its cardinal axes cuts, i.e. the range IPR and the azimuth IPR.

The fundamental shape of the IPR depends on the two-dimensional aperture that the data represents in the Fourier space of the target scene, modified by any aperture weighting employed, such as window functions prior to FFTs.

Anomalies in the IPR can point to likely faults in the radar hardware and/or software. A number of examples are discussed in this report. 
Education is when you read the fine print. Experience is what you get if you don't.

-- Pete Seeger 


\section{REFERENCES}

${ }^{1}$ F. M . Dickey, L. A. Romero, J. M. DeLaurentis, A. W. Doerry, "Window Functions for Imaging Radar: A Maximum Energy Approach to Contiguous and Notched Spectrums”, Optical Engineering, Vol. 42, No. 7, pp 2113-2128, July 2003.

2 A. W. Doerry, "Performance Limits for Synthetic Aperture Radar - second edition”, Sandia Report SAND2006-0821, Unlimited Release, February 2006.

3 A. W. Doerry, "SAR Processing with Non-Linear FM Chirp Waveforms”, Sandia Report SAND2006-7729, Unlimited Release, December 2006.

${ }^{4}$ Ronald Newbold Bracewell, The Fourier Transform and Its Application, Second Edition, ISBN 0-07-007013-X, McGraw-Hill, Inc., 1978.

${ }^{5}$ C. V. Jakowatz Jr., D. E. Wahl, P. H. Eichel, D. C. Ghiglia, P. A. Thompson, Spotlight-Mode Synthetic Aperture Radar: A Signal Processing Approach, ISBN 07923-9677-4, Kluwer Academic Publishers, 1996.

6 Merrill I. Skolnik, Introduction to Radar Systems, Second Edition, ISBN 0-07057909-1, McGraw-Hill, Inc., 1980.

7 J. W. Goodman, Introduction to Fourier Optics, McGraw-Hill, New York, 1968.

${ }^{8}$ F. M. Dickey, “Coherent optical simulation of radar imagery”, Optical Engineering, Vol. 15, No. 5, pp. 467-471, Sept/Oct. 1976.

9 J. T. Cordaro, "Range Performance of a Linear-FM Synthetic-Aperture Radar", Sandia Report SAND 87-1863, Official Use Only - Privileged Information (formerly Specified External Distribution Only), August 1987.

${ }^{10}$ A. W. Doerry, "Bandwidth requirements for fine resolution squinted SAR", SPIE 2000 International Symposium on Aerospace/Defense Sensing, Simulation, and Controls, Radar Sensor Technology V, Vol. 4033, Orlando FL, 27 April 2000.

11 J. T. Fuller, A. W. Doerry, “Transmitter Passband Requirements for SAR”, Sandia Report SAND2007-xxxx (to be published), 2007.

12 Alan V. Oppenheim, Ronald W. Schafer, Digital Signal Processing, ISBN 0-13214635-5, Prentice-Hall, 1975. 
${ }^{13}$ Peter A. Dudley, Bert L. Tise, "Multiplexed chirp waveform synthesizer”, US Patent 6,614,813, September 2, 2003.

${ }^{14}$ A. W. Doerry, “Autofocus Correction of Excessive Migration in Synthetic Aperture Radar Images”, Sandia Report SAND2004-4770, September 2004.

${ }^{15}$ A. W. Doerry, “Autofocus correction of SAR images exhibiting excessive residual migration”, SPIE 2005 Defense \& Security Symposium, Radar Sensor Technology X, Vol. 5788, Orlando FL, 28 March - 1 April 2005.

${ }^{16}$ Fred M. Dickey, Armin W. Doerry, Louis A. Romero, "Degrading effects of the lower atmosphere on long range airborne SAR imaging”, IET Proceedings on Radar, Sonar \& Navigation, Vol. 1, No. 5, pp. 329-339, 2007. 
It is impossible for a man to learn what he thinks he already knows.

-- Epictetus 


\section{DISTRIBUTION}

\begin{tabular}{|c|c|c|c|}
\hline \multicolumn{4}{|c|}{ Unlimited Release } \\
\hline 1 & MS 1330 & S. C. Holswade & 5340 \\
\hline 1 & MS 1330 & B. L. Burns & 5340 \\
\hline 1 & MS 0519 & T. J. Mirabal & 5341 \\
\hline 1 & MS 1330 & W. H. Hensley & 5342 \\
\hline 1 & MS 1330 & S. D. Bensonhaver & 5342 \\
\hline 1 & MS 1330 & T. P. Bielek & 5342 \\
\hline 1 & MS 1330 & A. W. Doerry & 5342 \\
\hline 1 & MS 1330 & D. W. Harmony & 5342 \\
\hline 1 & MS 1330 & J. A. Hollowell & 5342 \\
\hline 1 & MS 1330 & M. S. Murray & 5342 \\
\hline 1 & MS 1330 & S. Nance & 5342 \\
\hline 1 & MS 1330 & J. A. Rohwer & 5342 \\
\hline 1 & MS 1330 & D. G. Thompson & 5342 \\
\hline 1 & MS 0501 & P. R. Klarer & 5343 \\
\hline 1 & MS 1330 & K. W. Sorensen & 5345 \\
\hline 1 & MS 1330 & S. E. Allen & 5345 \\
\hline 1 & MS 0529 & B. C. Brock & 5345 \\
\hline 1 & MS 1330 & D. F. Dubbert & 5345 \\
\hline 1 & MS 1330 & G. R. Sloan & 5345 \\
\hline 1 & MS 1330 & S. M. Becker & 5348 \\
\hline 1 & MS 1330 & S. M. Devonshire & 5348 \\
\hline 1 & MS 1330 & M. W. Holzrichter & 5348 \\
\hline 1 & MS 1330 & O. M. Jeromin & 5348 \\
\hline 1 & MS 1330 & D. M. Small & 5348 \\
\hline 1 & MS 1330 & D. C. Sprauer & 5348 \\
\hline 1 & MS 1330 & A. D. Sweet & 5348 \\
\hline 1 & MS 0519 & D. L. Bickel & 5354 \\
\hline 1 & MS 0311 & F. M. Dickey & 2626 \\
\hline 2 & MS 9018 & Central Technical & \\
\hline 2 & MS 0899 & Technical Library & \\
\hline
\end{tabular}

8944

9536 